\title{
Viscosity solutions of path-dependent PDEs with randomized time
}

\author{
Zhenjie REN * $^{*} \quad$ Mauro Rosestolato ${ }^{\dagger}$
}

\begin{abstract}
We introduce a new definition of viscosity solution to path-dependent partial differential equations, which is a slight modification of the definition introduced in [8]. With the new definition, we prove the two important results till now missing in the literature, namely, a general stability result and a comparison result for semicontinuous sub-/super-solutions. As an application, we prove the existence of viscosity solutions using the Perron method. Moreover, we connect viscosity solutions of path-dependent PDEs with viscosity solutions of partial differential equations on Hilbert spaces.
\end{abstract}

Keywords: Viscosity solution; Path-dependent partial differential equations; Partial differential equations in infinite dimension.

AMS 2010 subject classification: 35K10; 35R15; 49L25; 60H30.

\section{Introduction}

This paper studies viscosity solutions of the fully nonlinear path-dependent partial differential equation

$$
-\partial_{t} u(t, \omega)-G\left(t, \omega, u(t, \omega), \partial_{\omega} u(t, \omega), \partial_{\omega \omega}^{2} u(t, \omega)\right)=0 \quad \text { on } \quad[0, T) \times \Omega .
$$

Here, $T>0$ is a given terminal time and $\omega \in \Omega$ is a continuous path from $[0, T]$ to $\mathbb{R}^{m}$ starting from the origin. The path derivatives $\partial_{t}, \partial_{\omega}, \partial_{\omega \omega}^{2}$ were first introduced in the work of Dupire [6]. See also [2] for the related Itô calculus. Such equations arise naturally in many applications. For example, the dynamic programming equation associated with a stochastic control problem of non-Markov diffusions (see [10]) and the one associated with a stochastic differential game with non-Markov dynamics (see [22]) both fall in the class of equation (1.1). The notion of nonlinear path-dependent partial differential equations was first proposed by Peng [19]. We also refer to Peng and Wang [20] for a study on classical solutions of semilinear equations.

The notion of viscosity solutions studied in this paper is a slight modification over the one introduced in Ekren et al. ([8]) in the semilinear context and further extended to the fully nonlinear case in $[10,11]$. Following the lines of the classical Crandall and Lions notion of viscosity solutions ([5]), supersolutions and subsolutions are defined through tangent test functions. However, while Crandall and Lions consider pointwise tangent functions, the tangency conditions in the path-dependent setting is in the sense of the expectation with respect to an appropriate class of probability measures $\mathscr{P}$. We refer to [27] for an overview, and to [3, 7, 14, 16, 23, 25, 26, 28] for some of the generalizations.

\footnotetext{
*Université Paris-Dauphine, PSL Research University, CNRS, UMR [7534], Ceremade, 75016 Paris, France, ren@ceremade.dauphine.fr.

${ }^{\dagger}$ CMAP, Ecole Polytechnique Paris, mauro.rosestolato@polytechnique.edu. Research supported by ERC 321111 Rofirm.

The authors are sincerely grateful to Nizar Touzi for valuable discussions.
} 
Regardless of the successful development mentioned above, there were some difficulties for the theory of viscosity solutions coming from the definition adopted in [8]. First, a good stability result was missing. In [10] the authors proved a stability result in the sense that if a sequence of uniformly continuous solutions $u^{n}$ uniformly converges to a uniformly continuous function $u$, then $u$ is also a solution. The assumptions of the uniform continuity and the uniform convergence are often too strong for applications, for example, for the Perron method to prove the existence of viscosity solution. Secondly, also a comparison result for semicontinuous viscosity sub-/supersolutions was missing. The aim of the present paper is to fill these two theoretical gaps. We do this by slightly modifying the definition in [8]. This modification is sufficient to let us overcome the technical difficulties for proving stability and comparison results, but does not compromise the other results till now obtained in the literature, such as existence.

In the previous definition, adopted in [8], the test function $\varphi$ is urged to be tangent to the (sub)solution $u$ at a point (say 0 ) in the sense that

$$
(u-\varphi)(0)=\max _{\tau \in \mathscr{T}} \sup _{\mathbb{P} \in \mathscr{P}} \mathbb{E}^{\mathbb{P}}[(u-\varphi)(\tau, \mathrm{B})],
$$

where $\mathscr{T}$ is the set of all stopping times taking values in $[0, T]$, and $\mathscr{P}$ is a family of probability measures on the path space $\Omega$ on which B is the canonical process. In the arguments for proving the stability and the comparison, we often need to solve the optimal stopping problem on the right hand side of (1.2), which is not a simple task and requires the uniform continuity of $u$ (see [9]). This turns out to be one of the main difficulties in improving the stability and the comparison results. In the present paper, in order to overcome this fundamental difficulty, we randomize the optimal stopping problem, and the new definition reads

$$
(u-\varphi)(0)=\sup _{\mathbb{P} \in \mathscr{P}} \mathbb{E}^{\mathbb{P}}[(u-\varphi)(\mathrm{T}, \mathrm{B})],
$$

where $\widetilde{\mathscr{P}}$ is a family of probability measures on the time-path product space $[0, T] \times \Omega$ on which $(T, B)$ is the canonical process. It turns out that the randomized problem can be solved for less regular functions $u$, and is more stable. With this change of definition, we manage to prove a general stability result for semicontinuous viscosity solutions (Theorem 4.5). The recipe of our proof is composed of the classical argument for stability in [4] and the measurable selection theorem. Moreover, by using the stability result, we are also able to prove the existence of viscosity solution through Perron's method (Theorem 5.3). Further, we prove a comparison result for semicontinuous sub-/super-solutions under some strong assumptions (Theorem 6.9), by approximating semicontinuous solutions with Lipschitz continuous solutions to approximating equations.

Another major contribution of this paper is to connect the path-dependent partial differential equation (1.1) with the partial differential equation

$$
-u_{t}-\left\langle A x, D_{x} u\right\rangle-G\left(t, x, u, D_{x_{0}} u, D_{x_{0} x_{0}}^{2} u\right)=0 \quad \text { on } \quad(0, T) \times H,
$$

where $H$ is a Hilbert space into which the path space $\Omega$ can embed, $A$ is an unbounded operator, and $D_{x_{0}}, D_{x_{0} x_{0}}^{2}$ are the first- and second-order differentials with respect to a finite dimensional subspace of $H$. Both equations (1.1) and (1.3) can be used to characterize the value function of non-Markov stochastic control problem. In this paper, we prove that a viscosity solution to the path-dependent equation (1.1), under some regularity assumption, is also a viscosity solution to the corresponding equation (1.3) (Theorem 7.6). The theory of viscosity solutions for partial differential equations on Hilbert spaces (we mainly refer to [12]) is designed of a large class of equations not rescrited to those of delay type, as (1.3). We notice that, till now, when applied to PDEs of the form (1.3), such a theory can deliver a comparison result under more general assumption on the nonlinearity function $G$, but only for more regular sub-/super-solutions. Moreover, the 
theory of path-dependent equations as here developed can treat solutions (semi)continuous in the $L^{\infty}$-norm, which cannot be settled in a Hilbert space framework.

The rest of the paper is organized as follows. Section 2 introduces the main notations. Section 3 presents the modified definition of the viscosity solutions to the path-dependent partial differential equations. In Section 4 we prove the stability result (Theorem 4.5), and using it in Section 5 we prove the existence of viscosity solution with Perron's method (Theorem 5.3). In Section 6 we show the comparison result for semicontinuous solutions (Theorem 6.9). In Section 7 we clarify the connection between the path-dependent equation and the correponding equation on the Hilbert space (Theorem 7.6). Finally, we complete some proofs in Appendix.

\section{Notations}

Canonical Space. Let $m>0$ be a natural number, $T>0$ be a real number. Define

$$
\Omega:=\left\{\omega \in C\left([0, T], \mathbb{R}^{m}\right): \omega(0)=0\right\} .
$$

We denote by $|\cdot|$ the Euclidean norm in $\mathbb{R}^{m}$ and by $|\cdot|_{\infty}$ the uniform norm on $\Omega$. In this paper, we study equations set on the spacetime space:

$$
\Theta:=[0, T] \times \Omega .
$$

For technical reasons, we also often work on the enlarged canonical space:

$$
\widetilde{\Theta}:=\Theta \times \Omega \times \Omega \times C_{0}\left([0, T], \mathbb{S}^{m}\right)
$$

where $\mathbb{S}^{m}$ is the space of symmetric $m \times m$ real matrices endowed with the supremum norm and

$$
C_{0}\left([0, T], \mathbb{S}^{m}\right):=\left\{f \in C\left([0, T], \mathbb{S}^{m}\right): f(0)=0\right\} .
$$

Hereafter, we will reserve the letter $\omega$ for a generic element of $\Omega$, the letter $\theta$ for the generic couple $\theta=(t, \omega) \in \Theta$, and the letter $\vartheta$ for the generic element $\vartheta=(\theta, a, \mu, q) \in \widetilde{\Theta}$. We introduce on $\Theta$ the pseudo-metric $d_{\infty}$ defined by

$$
d_{\infty}\left(\theta, \theta^{\prime}\right):=\left|t-t^{\prime}\right|+\left|\omega_{t \wedge \cdot}-\omega_{t^{\prime} \wedge} \cdot\right|_{\infty} .
$$

Remark 2.1. In this paper, without being otherwise stated, the (semi-)continuity on the canonical spaces is under $|\cdot|_{\infty}$. The (semi-)continuity related to other pseudo-metrics will be explicitly expressed.

Remark 2.2. Note the following facts:

- Let $\mathbb{F}:=\left\{\mathscr{F}_{t}\right\}_{t \in[0, T]}$ denote the canonical filtration on $\Omega$. All $d_{\infty}$-continuous functions are F-progressively measurable.

- $\lim _{n \rightarrow \infty}\left|\theta^{n}-\theta\right|_{\infty}=0$ implies that $\lim _{n \rightarrow \infty} d_{\infty}\left(\theta^{n}, \theta\right)=0$, and thus all $d_{\infty}$-continuous functions are continuous.

Let $\mathbb{E}^{\mathbb{S}}:=\left\{\mathscr{F}_{t}^{\mathbb{S}}\right\}_{t \in[0, T]}$ denote the canonical filtration on $C_{0}\left([0, T], \mathbb{S}^{m}\right)$. We introduce on $\widetilde{\Theta}$ the filtration $\mathbb{G}:=\left\{\mathscr{G}_{t}\right\}_{t \in[0, T]}$ defined by

$$
\mathscr{G}_{t}:=\left(\sigma\{[0, r], r \leq t\} \otimes \mathscr{F}_{t}\right) \otimes \mathscr{F}_{t} \otimes \mathscr{F}_{t} \otimes \mathscr{F}_{t}^{\mathbb{S}} \quad \forall t \in[0, T] .
$$


Shifted functions . For $\omega, \omega^{\prime} \in \Omega, t \in[0, T]$, we define

$$
\left(\omega \otimes_{t} \omega^{\prime}\right)_{s}:= \begin{cases}\omega_{s} & \text { if } s \in[0, t] \\ \omega_{t}+\omega_{s-t}^{\prime} & \text { if } s \in(t, T]\end{cases}
$$

For any function $\xi: \Omega \rightarrow R$ taking values in some set $R$, and for any $\theta=(t, \omega) \in \Theta$, we denote

$$
\xi^{\theta}\left(\omega^{\prime}\right):=\xi\left(\omega \otimes_{t} \omega^{\prime}\right) \quad \forall \omega^{\prime} \in \Omega .
$$

Similarly, given a function $u: \Theta \rightarrow R$, we denote, for $\theta \in \Theta$,

$$
u^{\theta}\left(t^{\prime}, \omega^{\prime}\right):=u\left(\left(t+t^{\prime}\right) \wedge T, \omega \otimes_{t} \omega^{\prime}\right) \quad \forall \theta^{\prime} \in \Theta .
$$

Clearly, if $\xi$ is $\mathscr{F}_{T}$-measurable then $\xi^{\theta}$ is $\mathscr{F}_{T-t}$-measurable, and if $X \mathbb{F}$-adapted then so is $X^{\theta}$.

Similarly, we can also shift functions defined on the enlarged canonical space $\widetilde{\Theta}$. For $t^{\prime} \in[0, T]$, $\theta \in \Theta, \vartheta \in \widetilde{\Theta}$, we denote

$$
\theta_{t^{\prime} \wedge \cdot}:=\left(t^{\prime} \wedge t, \omega_{t^{\prime} \wedge} .\right), \quad \vartheta_{t^{\prime} \wedge \cdot}=\left(\theta_{t^{\prime} \wedge \cdot,}, a_{t^{\prime} \wedge \cdot}, \mu_{t^{\prime} \wedge \cdot}, q_{t^{\prime} \wedge} .\right) .
$$

Given a function $v: \widetilde{\Theta} \rightarrow R$, we define for $\vartheta \in \widetilde{\Theta}$ and $s \in[0, T]$

$$
v^{s, \vartheta}\left(\vartheta^{\prime}\right):=v^{\vartheta_{s \wedge} \cdot}\left(\vartheta^{\prime}\right)=v\left(\left(t \wedge s+t^{\prime}\right) \wedge T, \omega \otimes_{t \wedge s} \omega^{\prime}, a \otimes_{t \wedge s} a^{\prime}, \mu \otimes_{t \wedge s} \mu^{\prime}, q \otimes_{t \wedge s} q^{\prime}\right) \quad \forall \vartheta^{\prime} \in \widetilde{\Theta} .
$$

Further, for a $\mathbb{G}$-stopping time $\tau$, we define

$$
v^{\tau, \vartheta}:=v^{\tau(\vartheta), \vartheta} .
$$

In particular, note that $v^{\tau, \vartheta}=v^{t \wedge \tau, \vartheta}$.

Probability Space. We denote by $\mathscr{P}$ the set of probability measures on $\left(\widetilde{\Theta}, \mathscr{G}_{T}\right)$. Unless otherwise specified, the set $\mathscr{P}$ is always endowed with the topology of the weak convergence. We recall that $\widetilde{\Theta}$ is a Polish space with respect to the product topology, hence $\mathscr{P}$ is a Polish space too. We denote by $T, B, A, M, Q$ the first, second, third, fourth, and fifth projection of $\widetilde{\Theta}$, respectively

$$
\begin{array}{r}
\mathrm{T}: \widetilde{\Theta} \rightarrow \mathbb{R}, \vartheta \mapsto t \quad \mathrm{~B}: \widetilde{\Theta} \rightarrow \Omega, \vartheta \mapsto \omega \quad \mathrm{A}: \widetilde{\Theta} \rightarrow \Omega, \vartheta \mapsto a \\
\mathrm{M}: \widetilde{\Theta} \rightarrow \Omega, \vartheta \mapsto \mu \quad \mathrm{Q}: \widetilde{\Theta} \rightarrow C_{0}\left([0, T], \mathbb{S}^{m}\right), \vartheta \mapsto q .
\end{array}
$$

We stress the fact that $T$ is a $\mathbb{G}$-stopping time and $B, A, M, Q$ are all $\mathbb{G}$-adapted processes. In order to simplify the notations, we also denote the quintuple ( $T, B, A, M, Q)$ by $X$,

$$
X:=(T, B, A, M, Q)
$$

Remark 2.3. Using the notations defined previously, we note that

$$
\mathrm{T}^{\tau, \vartheta}\left(\vartheta^{\prime}\right)=\left(t \wedge \tau(\vartheta)+t^{\prime}\right) \wedge T, \quad \mathrm{~B}^{\tau, \vartheta}\left(\vartheta^{\prime}\right)=\omega \otimes_{t \wedge \tau(\vartheta)} \omega^{\prime}, \quad \forall \vartheta, \vartheta^{\prime} \in \widetilde{\Theta},
$$

where $\tau$ is a $\mathbb{G}$-stopping time. Further, given a function $u: \Theta \rightarrow R$, we have

$$
u\left(\mathrm{~T}^{\tau, \vartheta}\left(\vartheta^{\prime}\right), \mathrm{B}^{\tau, \vartheta}\left(\vartheta^{\prime}\right)\right)=u\left(\left(t \wedge \tau(\vartheta)+t^{\prime}\right) \wedge T, \omega \otimes_{t \wedge \tau(\vartheta)} \omega^{\prime}\right)=u^{t \wedge \tau(\vartheta), \omega}\left(\theta^{\prime}\right)
$$

In this paper, we will use the following probability family on the enlarged canonical space $\widetilde{\Theta}$ to define the viscosity solutions to path-dependent PDEs. 
Definition 2.4. For $L>0$, we define the subset of $\widetilde{\Theta}$

$$
\widetilde{\Theta}_{L}:=\left\{\vartheta \in \widetilde{\Theta}: t \in[0, T], \omega=a+\mu, a, q \in A C\left([0, T], \mathbb{R}^{m}\right),|\dot{a}|_{\infty} \leq L,|\dot{q}|_{\infty} \leq L\right\},
$$

and the set of probabilities

$$
\mathscr{P}_{L}:=\left\{\mathbb{P} \in \mathscr{P}: \mathbb{P}\left(\widetilde{\Theta}_{L}\right)=1, \mathrm{M} \text { is a square-integrable } \mathbb{P} \text {-martingale, }\langle\mathrm{M}\rangle=\mathrm{Q} \mathbb{P} \text {-a.s. }\right\} .
$$

Using the canonical processes, we have for all $\mathbb{P} \in \mathscr{P}_{L}$ that

$$
\begin{gathered}
\mathrm{T} \in[0, T], \mathrm{B}=\mathrm{A}+\mathrm{M} \mathbb{P} \text {-a.s. } \\
\mathrm{M} \text { is a } \mathbb{P} \text {-martingale } \\
\langle\mathrm{M}\rangle=\mathrm{Q} \mathbb{P} \text {-a.s. } \\
\mathrm{A} \in A C\left([0, T], \mathbb{R}^{m}\right) \text { and }|\dot{\mathrm{A}}|_{\infty} \leq L \mathbb{P} \text {-a.s. } \\
\mathrm{Q} \in A C\left([0, T], \mathbb{R}^{m \times m}\right) \text { and }|\dot{\mathrm{Q}}|_{\infty} \leq L \mathbb{P} \text {-a.s. }
\end{gathered}
$$

Recall that $T$ is a $\mathbb{G}$-stopping time, so $B_{T \wedge \cdot}, A_{T \wedge \cdot,} M_{T \wedge}$. are $\mathbb{G}$-adapted and $M_{T \wedge}$. is a $\mathbb{P}$-martingale.

We introduce the sublinear and superlinear expectation operators associated with $\mathscr{P}_{L}$ :

$$
\overline{\mathscr{E}}_{L}:=\sup _{\mathbb{P} \in \mathscr{P}_{L}} \mathbb{E}^{\mathbb{P}} \quad \underline{\mathscr{E}}_{L}:=\inf _{\mathbb{P} \in \mathscr{P}_{L}} \mathbb{E}^{\mathbb{P}} .
$$

\section{Definition of $\mathscr{P}_{L}$-viscosity solution}

In this paper, we consider the fully nonlinear parabolic path-dependent PDE (PPDE):

$$
-\partial_{t} u-G\left(\theta, u, \partial_{\omega} u, \partial_{\omega \omega}^{2} u\right)=0 .
$$

In [27, 26], it is showed that one can define viscosity solutions for PPDEs via jets. In this manuscript, we start directly from the definition via jets. For $\alpha \in \mathbb{R}, \beta \in \mathbb{R}^{m}, \gamma \in \mathbb{S}^{m}$, let

$$
\varphi^{\alpha, \beta, \gamma}(\theta):=\alpha t+\left\langle\beta, \omega_{t}\right\rangle+\frac{1}{2}\left\langle\gamma \omega_{t}, \omega_{t}\right\rangle \quad \forall \theta \in \Theta,
$$

where $\langle\cdot, \cdot\rangle$ denotes the standard scalar product on $\mathbb{R}^{m}$.

A further tool for the type of localization that we will implement in the definition of viscosity solution if the function $\mathrm{H}_{\delta}$. For any $\delta \in(0, T]$, define the function $\mathrm{H}_{\delta}: \widetilde{\Theta} \rightarrow[0, T]$ by

$$
\mathrm{H}_{\delta}(\vartheta):=\inf \left\{s>0: d_{\infty}((s, \omega), 0)=s+\left|\omega_{s \wedge} \cdot\right|_{\infty} \geq \delta\right\} .
$$

It is not difficult to show that $\mathrm{H}_{\delta}$ is continuous.

Let $\theta \in \Theta$, with $t<T$. For $u: \Theta \rightarrow \mathbb{R}$ upper semicontinuous, locally bounded from above, the subjet of $u$ in $\theta$ is defined by

$$
\underline{\mathscr{J}}_{L} u(\vartheta):=\left\{(\alpha, \beta, \gamma) \in \mathbb{R} \times \mathbb{R}^{m} \times \mathbb{S}^{m}: u(\theta)=\overline{\mathscr{E}}_{L}\left[\left(u^{\theta}-\varphi^{\alpha, \beta, \gamma}\right)\left(\mathrm{T} \wedge \mathrm{H}_{\delta}, \mathrm{B}\right)\right] \text { for some } \delta \in(0, T-t]\right\},
$$

In a symmetric way, for a lower semicontinuous function $u: \Theta \rightarrow \mathbb{R}$, locally bounded from below, the superjet of $u$ in $\theta$ is defined by

$$
\overline{\mathscr{J}}_{L} u(\theta):=\left\{(\alpha, \beta, \gamma) \in \mathbb{R} \times \mathbb{R}^{m} \times \mathbb{S}^{m}: u(\theta)=\underline{\mathscr{E}}_{L}\left[\left(u^{\theta}-\varphi^{\alpha, \beta, \gamma}\right)\left(\mathrm{T} \wedge \mathrm{H}_{\delta}, \mathrm{B}\right)\right] \text { for some } \delta \in(0, T-t]\right\} .
$$

We denote $\overline{\mathbb{R}}:=\mathbb{R} \cup\{-\infty,+\infty\}$. 
Definition 3.1 ( $\mathscr{P}_{L}$-viscosity sub-/supersolution). Let $G: \Theta \times \mathbb{R} \times \mathbb{R}^{m} \times \mathbb{S}^{m} \rightarrow \overline{\mathbb{R}}$ be a function. A $d_{\infty}$-upper semicontinuous (resp. $d_{\infty}$-lower semicontinuous) function $u: \Theta \rightarrow \mathbb{R}$, locally bounded from above (resp. locally bounded from below) is a $\mathscr{P}_{L}$-viscosity subsolution (resp. $\mathscr{P}_{L}$-viscosity supersolution) of (3.1) if

$$
\begin{array}{cc}
-\alpha-G(\theta, u(\theta), \beta, \gamma) \leq 0 & \forall \theta \in \Theta,(\alpha, \beta, \gamma) \in \mathscr{\mathscr { J }}_{L} u(\theta), \\
\text { (resp. }-\alpha-G(\theta, u(\theta), \beta, \gamma) \geq 0 & \left.\forall \theta \in \Theta,(\alpha, \beta, \gamma) \in \overline{\mathscr{J}}_{L} u(\theta)\right) .
\end{array}
$$

A locally bounded continuous function $u$ is a $\mathscr{P}_{L}$-viscosity solution of (3.1) if it is both a $\mathscr{P}_{L^{-}}$ viscosity sub- and supersolution of (3.1).

Remark 3.2. Let us recall the previous definition of viscosity solution of path-dependent PDEs. Define the probability family $\mathscr{P}_{L}^{\prime}$ on the space $\widetilde{\Theta}^{\prime}:=\Omega \times \Omega \times \Omega \times C_{0}\left([0, T], \mathbb{S}^{m}\right)$ (we still use the notations of canonical processes $B, A, M, Q)$ :

$$
\begin{aligned}
\mathscr{P}_{L}^{\prime}:=\{\mathbb{P}: \mathrm{B}=\mathrm{A}+\mathrm{M}, \mathrm{M} \text { is a } \mathbb{P} \text {-martingale, } & \langle\mathrm{M}\rangle=\mathrm{Q}, \mathrm{A} \in A C\left([0, T], \mathbb{R}^{m}\right), \\
& \left.\mathrm{Q} \in A C\left([0, T], \mathbb{R}^{m, m}\right),|\dot{A}|_{\infty} \leq L,|\dot{Q}|_{\infty} \leq L, \mathbb{P} \text {-a.s. }\right\}
\end{aligned}
$$

Note that the space $\widetilde{\Theta}^{\prime}$ misses the time dimension, compared to the canonical space $\widetilde{\Theta}$ in the present paper. Define the nonlinear expectation $\overline{\mathscr{E}}_{L}^{\prime}:=\sup _{\mathbb{P} \in \mathscr{P}_{L}^{\prime}} \mathbb{E}^{\mathbb{P}}$ and the subjet

$$
\underline{\mathscr{J}}_{L}^{\prime} u(\theta):=\left\{(\alpha, \beta, \theta) \in \mathbb{R} \times \mathbb{R}^{m} \times \mathbb{S}^{m}: u(\theta)=\max _{\tau \in \mathscr{T}} \overline{\mathscr{E}}_{L}^{\prime}\left[\left(u^{\theta}-\varphi^{\alpha, \beta, \theta}\right)\left(\tau \wedge \mathrm{H}_{\delta}, \mathrm{B}\right)\right] \text { for some } \delta \in(0, T-t]\right\},
$$

where $\mathscr{T}$ is the set of all $\mathbb{F}^{\mathrm{B}}$-stopping times. Similarly we can define the superjet $\overline{\mathscr{J}}_{L}^{\prime}$. Then we define $\mathscr{P}_{L}^{\prime}$-viscosity solutions as in Definition 3.1 , by replacing the jets $\overline{\mathscr{J}}_{L}, \underline{\mathscr{J}}_{L}$ by $\frac{\mathscr{J}}{L}_{L}^{\prime}, \underline{\mathscr{J}}_{L}^{\prime}$. As we see, in our new definition of subjet the function $(u-\varphi)_{\mathrm{H}_{\delta} \wedge}^{\theta}$. reaches its maximum at 0 in the sense of $\overline{\mathscr{E}}_{L}$ instead of $\max _{\tau \in \mathscr{T}} \overline{\mathscr{E}}_{L}^{\prime}$. By doing so, the maximization over the stopping times $\tau \in \mathscr{T}$ is replaced by the maximization of the laws applied on the canonical variable $T$. Indeed, the new nonlinear expectation $\overline{\mathscr{E}}_{L}$ is a randomized optimal stopping operator. In general, one need fewer assumptions to ensure the existence of

$$
\mathbb{P}^{*} \in \underset{\mathbb{P} \in \mathscr{P}_{L}}{\arg \max _{L}} \mathbb{E}^{\mathbb{P}}[f] \quad \text { than that of } \quad \tau^{*} \in \underset{\tau \in \mathscr{T}}{\arg \max } \overline{\mathscr{E}}_{L}^{\prime}[f], \quad \text { for } f: \Theta \rightarrow \mathbb{R}
$$

In particular, the optimal probability $\mathbb{P}^{*}$ for the first optimization exists if $f$ is $d_{\infty}$-u.s.c. and bounded from above (see Section A), while in [9] the authors proved for the second optimization the optimal stopping time $\tau^{*}$ exists if $f$ is bounded $d_{\infty}$-uniformly continuous. This change allows the viscosity solution under the new definition to have better properties. For example, it allows us to prove a stronger stability of solutions (see Section 4) and a comparison result for semicontinuous solutions (see Section 6).

Finally, the change of definition does not threat the already proven results in the pathdependant PDE literature, namely, comparison [11, 26, 28], convergence of numerical schemes [25], etc. In fact, the arguments for these results will stay in the same lines, while necessary modifications need to be made concerning the optimization problems in (3.4).

\section{Stability}

For all $\mathbb{G}$-stopping time $\tau \leq T$, define the family:

$$
\mathscr{P}_{L}(\tau, \vartheta):=\left\{\mathbb{P} \in \mathscr{P}_{L}: T \leq T-\tau(\vartheta), \mathbb{P} \text {-a.s. }\right\} .
$$


We list the following important properties concerning the families of probabilities $\mathscr{P}_{L}$ and $\mathscr{P}_{L}(\tau, \vartheta)$. The proofs are postponed to Appendix.

Proposition 4.1. The set $\mathscr{P}_{L}$ is compact.

Lemma 4.2. Let $\tau: \widetilde{\Theta} \rightarrow[0, T]$ be a continuous $\mathbb{G}$-stopping time. Then the graph of the function

$$
\widetilde{\Theta} \rightarrow \mathscr{P}, \vartheta \mapsto \mathscr{P}_{L}(\tau, \vartheta)
$$

is closed, i.e., given $\left\{\left(\vartheta^{n}, \mathbb{P}^{n}\right)\right\}_{n \in \mathbb{N}},\left(\vartheta^{*}, \mathbb{P}^{*}\right)$ such that $\mathbb{P}^{n} \in \mathscr{P}_{L}\left(\tau, \vartheta^{n}\right)$ for each $n,\left|\vartheta^{n}-\vartheta^{*}\right|_{\infty} \rightarrow 0$ and $\mathbb{P}^{n} \rightarrow \mathbb{P}^{*}$, we have $\mathbb{P}^{*} \in \mathscr{P}_{L}\left(\tau, \vartheta^{*}\right)$.

Define the nonlinear (conditional) expectations:

$$
\overline{\mathscr{E}}_{L}^{\tau}[\cdot](\vartheta):=\sup _{\mathbb{P} \in \mathscr{P}_{L}(\tau, \vartheta)} \mathbb{E}^{\mathbb{P}}[\cdot] \quad \underline{\mathscr{E}}_{L}^{\tau}[\cdot](\vartheta):=\inf _{\mathbb{P} \in \mathscr{P}_{L}(\tau, \vartheta)} \mathbb{E}^{\mathbb{P}}[\cdot]
$$

Proposition 4.3. Let $f: \Theta \rightarrow \mathbb{R}$ be $d_{\infty}$-u.s.c. bounded from above, and $\tau$ be a continuous $\mathbb{G}$-stopping time. We have

$$
\overline{\mathscr{E}}_{L}\left[f 1_{\{\mathrm{T}<\tau\}}+\overline{\mathscr{E}}_{L}^{\tau}\left[f^{\theta}\right]_{\mid \theta=(\tau, \mathrm{B})} 1_{\{\tau \leq \mathrm{T}\}}\right]=\overline{\mathscr{E}}_{L}[f] .
$$

In particular we have

$$
\overline{\mathscr{E}}_{L}\left[\overline{\mathscr{E}}_{L}^{\mathrm{T}}\left[f^{\theta}\right]_{\mid \theta=(\mathrm{T}, \mathrm{B})}\right]=\overline{\mathscr{E}}_{L}[f] .
$$

Moreover, let $\mathbb{P}^{*} \in \mathscr{P}_{L}$ be such that $\overline{\mathscr{E}}_{L}[f]=\mathbb{E}^{\mathbb{P}^{*}}[f]$. Then we have

$$
f=\overline{\mathscr{E}}_{L}^{\top}\left[f^{\top, \mathrm{B}}\right] \quad \mathbb{P}^{*} \text {-a.s. }
$$

As explained in Remark 3.2, in this section we will exploit the advantage of the new definition of the jets in order to prove a better stability result. As we will see in the rest of the paper, one may apply many pseudo-metrics on $\Theta$ other than $d_{\infty}$.

In what follows, we denote by $d$ a pseudo-metric on $\Theta$ which is $d_{\infty}$-continuous and such that $d\left(\theta, \theta^{\prime}\right)=0$ implies $t=t^{\prime}$ and $\omega_{t \wedge \cdot}=\omega_{t \wedge}^{\prime}$. In order to recall that $d$ has these properties, we will often write $d \ll d_{\infty}$.

Proposition 4.4. Let $d$ be a pseudo-metric defined on $\Theta$ such that $d \ll d_{\infty}$. Let $u_{n}$ be a sequence of $d_{\infty}$-u.s.c. functions uniformly bounded from above. Define the function $u: \Theta \rightarrow \mathbb{R}$ by

$$
u(\theta):=\limsup _{\substack{d(\hat{\theta}, \theta) \rightarrow 0 \\ n \rightarrow \infty}} u_{n}(\hat{\theta})
$$

Then, for any $(\alpha, \beta, \gamma) \in \underline{\mathscr{J}}_{L} u(\theta)$, there exist $\hat{\theta}_{n} \in \Theta$ and $\left(\alpha_{n}, \beta_{n}, \gamma\right) \in \underline{\mathscr{J}}_{L} u_{n}\left(\hat{\theta}_{n}\right)$ such that

$$
d\left(\hat{\theta}_{n}, \theta\right) \rightarrow 0, \quad\left(u_{n}\left(\hat{\theta}_{n}\right), \alpha_{n}, \beta_{n}\right) \rightarrow(u(\theta), \alpha, \beta) .
$$

Proof. Step 1. Since $(\alpha, \beta, \gamma) \in \underline{\mathscr{J}}_{L} u(\theta)$, we also have $(\alpha+\varepsilon, \beta, \gamma) \in \underline{\mathscr{J}}_{L} u(\theta)$ for any $\varepsilon>0$. Let $\theta_{n}$ be a sequence such that

$$
d\left(\theta_{n}, \theta\right) \rightarrow 0 \quad \text { and } \quad u(\theta)=\lim _{n \rightarrow \infty} u_{n}\left(\theta_{n}\right) .
$$

For the simplicity of notation, we denote

$$
U\left(\theta^{\prime}\right):=\left(u^{\theta}-\varphi^{\alpha+\varepsilon, \beta, \gamma}\right)\left(t^{\prime} \wedge \mathrm{H}_{\delta}\left(\theta^{\prime}\right), \omega^{\prime}\right), \quad U_{n}\left(\theta^{\prime}\right):=\left(u_{n}^{\theta_{n}}-\varphi^{\alpha+\varepsilon, \beta, \gamma}\right)\left(t^{\prime} \wedge \mathrm{H}_{\delta}\left(\theta^{\prime}\right), \omega^{\prime}\right), \quad \text { for all } \theta^{\prime} \in \Theta .
$$


Since $\mathscr{P}_{L}$ is compact (Proposition 4.1) and the maps $\mathscr{P} \rightarrow \overline{\mathbb{R}}, \mathbb{P} \mapsto \mathbb{E}^{\mathbb{P}}\left[U_{n}\right]$ are upper semicontinuous, there exists $\mathbb{P}_{n} \in \mathscr{P}_{L}$ for each $n \in \mathbb{N}$ such that

$$
\mathbb{E}^{\mathbb{P}_{n}}\left[U_{n}\right]=\overline{\mathscr{E}}_{L}\left[U_{n}\right]
$$

By considering a subsequence if necessary, again denoted by $\mathbb{P}_{n}$, we can find $\mathbb{P}^{*} \in \mathscr{P}_{L}$ such that $\mathbb{P}_{n} \rightarrow \mathbb{P}^{*}$. By Skorohod's representation there exists a probability space $(\widehat{\Omega}, \widehat{\mathscr{G}}, \widehat{\mathbb{P}})$ on which

$\exists \quad$ r.v.'s $\left.\left.\quad \mathrm{X}^{n}\right|_{\widehat{\mathbb{P}}} \stackrel{d}{=} \mathrm{X}\right|_{\mathbb{P}_{n}},\left.\left.\quad \mathrm{X}^{*}\right|_{\widehat{\mathbb{P}}} \stackrel{d}{=} \mathrm{X}\right|_{\mathbb{P}^{*}}$ such that $\left|\mathrm{X}^{n}-\mathrm{X}^{*}\right|_{\infty} \rightarrow 0 \quad \widehat{\mathbb{P}}$-a.s.,

where $\stackrel{d}{=}$ denotes equality in distribution. Together with (4.3) we obtain

$$
\begin{aligned}
u(\theta) & =\lim _{n \rightarrow \infty} u_{n}\left(\theta_{n}\right) \leq \limsup _{n \rightarrow \infty} \overline{\mathscr{E}}_{L}\left[U_{n}\right]=\limsup _{n \rightarrow \infty} \mathbb{E}^{\mathbb{P}_{n}}\left[U_{n}\right]=\limsup _{n \rightarrow \infty} \mathbb{E}^{\widehat{P}}\left[U_{n}\left(\mathrm{X}^{n}\right)\right] \\
& \leq \mathbb{E}^{\widehat{\mathbb{P}}}\left[\limsup _{n \rightarrow \infty} U_{n}\left(\mathrm{X}^{n}\right)\right] \leq \mathbb{E}^{\widehat{\mathbb{P}}}\left[U\left(\mathrm{X}^{*}\right)\right]=\mathbb{E}^{\mathbb{P}^{*}}[U] \leq \overline{\mathscr{E}}_{L}[U]=u(\theta),
\end{aligned}
$$

The first inequality in the last line is due to Fatou's lemma, the second one is due to the definition of $u$, whereas the last equality is due to the assumption $(\alpha, \beta, \gamma) \in \underline{\mathcal{J}}_{L} u(\theta)$ and by choosing any $\delta$ sufficiently small. Therefore

$$
\mathbb{E}^{\mathbb{P}^{*}}[U]=\overline{\mathscr{E}}_{L}[U]
$$

which, together with $(\alpha, \beta, \gamma) \in \overline{\mathscr{J}}_{L} u(\theta)$, provides

$$
\mathbb{P}^{*}[\mathrm{~T}=0]=1 .
$$

Since $\mathrm{H}_{\delta}$ is continuous, from (4.6) and by denoting $\mathrm{T}^{*}=\mathrm{T}\left(\mathrm{X}^{*}\right), \mathrm{T}^{n}=\mathrm{T}\left(\mathrm{X}^{n}\right)$, we have

$$
1=\widehat{\mathbb{P}}\left[\mathrm{T}^{*}=0\right]=\widehat{\mathbb{P}}\left[\mathrm{T}^{*}<\mathrm{H}_{\delta}\left(\mathrm{X}^{*}\right)\right]=\widehat{\mathbb{P}}\left[\lim _{n \rightarrow \infty}\left(\mathrm{T}^{n}-\mathrm{H}_{\delta}\left(\mathrm{X}^{n}\right)\right)<0\right] \leq \widehat{\mathbb{P}}\left[\liminf _{n \rightarrow \infty}\left\{\mathrm{T}^{n}<\mathrm{H}_{\delta}\left(\mathrm{X}^{n}\right)\right\}\right] .
$$

Further, denoting $\mathrm{B}^{*}=\mathrm{B}\left(\mathrm{X}^{*}\right)$ and $\mathrm{B}^{n}=\mathrm{B}\left(\mathrm{X}^{n}\right)$, by (4.4),(4.6) and by $d_{\infty}$-continuity of $d$, we have

$$
\begin{aligned}
1 & =\widehat{\mathbb{P}}\left[d\left(\theta,\left(t+\mathrm{T}^{*}, \omega \otimes_{t} \mathrm{~B}^{*}\right)\right)<\delta\right]=\widehat{\mathbb{P}}\left[\lim _{n \rightarrow \infty} d\left(\theta_{n},\left(t_{n}+\mathrm{T}^{n}, \omega_{n} \otimes_{t_{n}} \mathrm{~B}^{n}\right)\right)<\delta\right] \\
& \leq \widehat{\mathbb{P}}\left[\liminf _{n \rightarrow \infty}\left\{d\left(\theta_{n},\left(t_{n}+\mathrm{T}^{n}, \omega_{n} \otimes_{t_{n}} \mathrm{~B}^{n}\right)\right)<\delta\right\}\right] .
\end{aligned}
$$

Step 2. It follows from (4.3) and Proposition 4.3 that

$$
U_{n}=\overline{\mathscr{E}}_{L}^{\top}\left[U_{n}^{\top, \mathrm{B}}\right], \quad \mathbb{P}_{n} \text {-a.s. }
$$

Therefore, for all $n \in \mathbb{N}$,

$$
\mathbb{P}_{n}\left[\Xi^{n}\right]=1, \quad \text { where } \quad \Xi^{n}:=\left\{\vartheta^{\prime} \in \widetilde{\Theta}: U_{n}\left(\theta^{\prime}\right)=\overline{\mathscr{E}}_{L}^{t^{\prime}}\left[U_{n}^{\theta^{\prime}}\right]\left(\vartheta^{\prime}\right)\right\} .
$$

Note that

$$
\Xi^{n} \cap\left\{\mathrm{T}<\mathrm{H}_{\delta}\right\} \subset\left\{\vartheta^{\prime} \in \widetilde{\Theta}:\left(\alpha+\varepsilon, \beta+2 \gamma \omega_{t^{\prime}}^{\prime}, \gamma\right) \in \underline{\mathscr{J}}_{L} u_{n}^{\theta_{n}}\left(\theta^{\prime}\right)\right\},
$$

and by (4.7), (4.8) and (4.9) we have

$$
\widehat{\mathbb{P}}\left[\liminf _{n \rightarrow \infty}\left\{\left(\alpha+\varepsilon, \beta+2 \gamma \mathrm{B}_{\mathrm{T}^{n}}^{n}, \gamma\right) \in \underline{\mathcal{J}}{ }_{L} u_{n}^{\theta_{n}}\left(\mathrm{~T}^{n}, \mathrm{~B}^{n}\right), d\left(\theta_{n},\left(t_{n}+\mathrm{T}^{n}, \omega_{n} \otimes_{t_{n}} \mathrm{~B}^{n}\right)\right)<\delta\right\}\right]=1 .
$$

Step 3. It follows from (4.6) that

$$
\widehat{\mathbb{P}}\left[\lim _{n \rightarrow \infty} \mathrm{T}^{n}=0\right]=1
$$


Together with (4.5) we obtain

$$
u(\theta)=\mathbb{E}^{\widehat{P}}\left[\limsup _{n \rightarrow \infty} U_{n}\left(\mathrm{X}^{n}\right)\right]=\mathbb{E}^{\widehat{P}}\left[\limsup _{n \rightarrow \infty} u_{n}^{\theta_{n}}\left(\mathrm{~T}^{n}, \mathrm{~B}^{n}\right)\right] .
$$

By the definition of $u$ we know that $\lim \sup _{n \rightarrow \infty} u_{n}^{\theta_{n}}\left(\mathrm{~T}^{n}, \mathrm{~B}^{n}\right) \leq u(\theta) \widehat{\mathbb{P}}$-a.s. Therefore,

$$
\widehat{\mathbb{P}}\left[\limsup _{n \rightarrow \infty} u_{n}^{\theta_{n}}\left(\mathrm{~T}^{n}, \mathrm{~B}^{n}\right)=u(\theta)\right]=1 \text {. }
$$

Together with (4.10), we obtain

$$
\begin{aligned}
& \widehat{\mathbb{P}}\left[\left\{\limsup _{n \rightarrow \infty} u_{n}^{\theta_{n}}\left(\mathrm{~T}^{n}, \mathrm{~B}^{n}\right)=u(\theta)\right\}\right. \\
&\left.\quad \cap \liminf _{n \rightarrow \infty}\left\{\left(\alpha+\varepsilon, \beta+2 \gamma \mathrm{B}_{\mathrm{T}^{n}}^{n}, \gamma\right) \in \underline{\mathscr{J}}_{L} u_{n}^{\theta_{n}}\left(\mathrm{~T}^{n}, \mathrm{~B}^{n}\right), d\left(\theta_{n},\left(t_{n}+\mathrm{T}^{n}, \omega_{n} \otimes_{t_{n}} \mathrm{~B}^{n}\right)\right)<\delta\right\}\right]=1 .
\end{aligned}
$$

Denote $\hat{\theta}_{n}:=\left(t_{n}+\mathrm{T}^{n}\left(\vartheta^{\prime}\right), \omega_{n} \otimes_{t_{n}} \mathrm{~B}^{n}\left(\vartheta^{\prime}\right)\right)$ and $\beta_{n}:=\beta+2 \gamma \mathrm{B}_{\mathrm{T}^{n}}^{n}\left(\vartheta^{\prime}\right)$ for all $\vartheta^{\prime} \in \widetilde{\Theta}, n \in \mathbb{N}$. It follows from (4.11) that there exist $\vartheta^{\prime}$ and $n$ such that

$$
d\left(\hat{\theta}_{n}, \theta\right)<d\left(\theta_{n}, \theta\right)+\delta, \quad\left|u_{n}\left(\hat{\theta}_{n}\right)-u(\theta)\right|<\delta, \quad\left|\beta_{n}-\beta\right|<2 \gamma \delta, \quad \text { and } \quad\left(\alpha+\varepsilon, \beta_{n}, \gamma\right) \in \underline{\mathscr{J}}_{L} u_{n}\left(\hat{\theta}_{n}\right) .
$$

Finally, since the constants $\delta, \varepsilon$ can be chosen arbitrarily small, the desired result follows.

As direct consequence of Proposition 4.4 we have the following

Theorem 4.5. Let $d, u_{n}, u$ be as in Proposition 4.4. Let $G_{n}: \Theta \times \mathbb{R} \times \mathbb{R}^{m} \times \mathbb{S}^{m} \rightarrow \overline{\mathbb{R}}$ be a sequence of functions. Define $G: \Theta \times \mathbb{R} \times \mathbb{R}^{m} \times \mathbb{S}^{m} \rightarrow \overline{\mathbb{R}}$ by

$$
G(\theta, r, \beta, \gamma):=\limsup _{\substack{n \rightarrow \infty, d\left(\theta_{n}, \theta\right) \rightarrow 0 \\ r_{n} \rightarrow r, \beta_{n} \rightarrow \beta}} G_{n}\left(\theta_{n}, r_{n}, \beta_{n}, \gamma\right)
$$

If $u_{n}$ is a $\mathscr{P}_{L}$-viscosity subsolution of

$$
-\partial_{t} u_{n}-G_{n}\left(\theta, u_{n}, \partial_{\omega} u_{n}, \partial_{\omega \omega}^{2} u_{n}\right)=0,
$$

then $u$ is a $\mathscr{P}_{L}$-viscosity subsolution of

$$
-\partial_{t} u-G\left(\theta, u, \partial_{\omega} u, \partial_{\omega \omega}^{2} u\right)=0 .
$$

\section{Existence of $\mathscr{P}_{L}$-viscosity solution: Perron's method}

As we see in the classical literature on the viscosity solutions, one can apply the stability and the comparison results for semi-continuous solutions to prove the existence of viscosity solution via the so-called Perron's method. In Section 4, we have established a quite general stability result (Theorem 4.5), and we will leave the discussion on the comparison result to the next sections. In this section, we adapt the Perron's method to the context of $\mathscr{P}_{L}$-viscosity solution, assuming some comparison result holds true.

Assumptions 5.1. Let $d$ be a pseudo-metric on $\Theta$ such that $d \ll d_{\infty}$.

(i) $G: \Theta \times \mathbb{R} \times \mathbb{R}^{m} \times \mathbb{S}^{m} \rightarrow \mathbb{R}$ is $d+|\cdot|+|\cdot|_{\infty}+|\cdot|_{\infty}$-uniformly continuous on $d+|\cdot|+|\cdot|_{\infty}+|\cdot|_{\infty}$-bounded sets.

(ii) For every $(\theta, \beta, \gamma) \in \Theta \times \mathbb{R}^{m} \times \mathbb{S}^{m}$, the function $r \mapsto G(\theta, r, \beta, \gamma)$ is non-decreasing. 
(iii) For every $(\theta, r, \beta) \in \Theta \times \mathbb{R} \times \mathbb{R}^{m}$,

$$
G(\theta, r, \beta, \gamma) \leq G\left(\theta, r, \beta, \gamma^{\prime}\right) \quad \forall \gamma, \gamma^{\prime} \in \mathbb{S}^{m}, \gamma \leq \gamma^{\prime} .
$$

(iv) For all $(\theta, r) \in \Theta \times \mathbb{R}, \beta, \beta^{\prime} \in \mathbb{R}^{m}, \gamma, \gamma^{\prime} \in \mathbb{S}^{m}$,

$$
\left|G\left(\theta, r, \beta+\beta^{\prime}, \gamma+\gamma^{\prime}\right)-G(\theta, r, \beta, \gamma)\right| \leq L\left(\left|\beta^{\prime}\right|+\left|\gamma^{\prime}\right|\right) .
$$

Assumption 5.2. Let $d$ be a pseudo-metric on $\Theta$ such that $d \ll d_{\infty}$. Assume that there is a bounded $d$-u.s.c. viscosity subsolution $\underline{u}$ and a bounded $d$-l.s.c. viscosity supersolution $\bar{v}$ of PPDE (3.1) such that

i) $\underline{u} \leq \bar{v}$ on $\Theta$; if $\underline{u}_{*}$ denote the $d$-l.s.c. envelop of $\underline{u}$, then $\underline{u}_{*}(T, \cdot)=\bar{v}(T, \cdot)=: \xi$.

ii) For any $d$-u.s.c. viscosity subsolution $u$ and $d$-l.s.c. viscosity supersolution $v$ of PPDE (3.1) taking values between $\underline{u}_{*}$ and $\bar{v}$, we have $u \leq v$ on $\Theta$.

Theorem 5.3. Let Assumptions 5.1 and 5.2 hold true. Denote

$\mathscr{D}:=\left\{\phi: \Theta \rightarrow \mathbb{R}: \phi\right.$ is a bounded d-u.s.c. $\mathscr{P}_{L}$-viscosity subsolution of (3.1) and $\left.\underline{u} \leq \phi \leq \bar{v}\right\}$.

Then $u(\theta):=\sup \{\phi(\theta): \phi \in \mathscr{D}\}$ is a d-continuous $\mathscr{P}_{L}$-viscosity solution of (3.1), and satisfies the boundary condition $u(T, \cdot)=\xi$.

Before proving Theorem 5.3, we show some useful lemmas. The following proposition is a direct corollary of the stability result in Proposition 4.4.

Proposition 5.4. Let $\bar{u}$ be $d_{\infty}$-u.s.c., $\theta \in \Theta$ and $(\alpha, \beta, \gamma) \in \underline{J}_{L} \bar{u}(\theta)$. Suppose also that $u_{n}$ is a sequence of $d_{\infty}$-u.s.c. functions uniformly bounded from above such that

$$
\left\{\begin{array}{l}
(1) \text { there exist } \theta_{n} \in \Theta \text { such that } d\left(\theta_{n}, \theta\right) \rightarrow 0, u_{n}\left(\theta_{n}\right) \rightarrow \bar{u}(\theta), \\
(2) \text { if } \theta_{n}^{\prime} \in \Theta \text { and } d\left(\theta_{n}^{\prime}, \theta^{\prime}\right) \rightarrow 0 \text {, then } \limsup _{n \rightarrow \infty} u_{n}\left(\theta_{n}^{\prime}\right) \leq \bar{u}\left(\theta^{\prime}\right) .
\end{array}\right.
$$

Then there exist $\hat{\theta}_{n} \in \Theta,\left(\alpha_{n}, \beta_{n}, \gamma\right) \in \underline{\mathscr{J}}_{L} u_{n}\left(\hat{\theta}_{n}\right)$ such that

$$
d\left(\hat{\theta}_{n}, \theta\right) \rightarrow 0, \quad\left(u_{n}\left(\hat{\theta}_{n}\right), \alpha_{n}, \beta_{n}\right) \rightarrow(\bar{u}(\theta), \alpha, \beta) .
$$

Proof. Recall the definition of $u$ in Proposition 4.4. We clearly have

$$
\bar{u}(\theta)=u(\theta) \text { and } \bar{u}\left(\theta^{\prime}\right) \geq u\left(\theta^{\prime}\right) \text { for all } \theta^{\prime} \in \Theta
$$

It the follows that $(\alpha, \beta, \gamma) \in \underline{\mathscr{J}}_{L} u(\theta)$. Then the desired result follows from Proposition 4.4.

For $\gamma \in \mathbb{S}^{m}$, we define

$$
(\gamma)^{+}:=\sup _{x \in \mathbb{R}^{m},|x| \leq 1}\langle\gamma x, x\rangle
$$

Lemma 5.5. Let $G$ as in Assumption 5.1. Then, for all $\theta \in \Theta, r \in \mathbb{R}, \beta \in \mathbb{R}^{m}, \gamma, \gamma^{\prime} \in \mathbb{S}^{m}$,

$$
G(\theta, r, \beta, \gamma)-G\left(\theta, r, \beta, \gamma^{\prime}\right) \leq\left(\gamma-\gamma^{\prime}\right)^{+}
$$


Proof. By definition of (. $)^{+}$in (5.1), we have $\gamma-\gamma^{\prime} \leq L\left(\gamma-\gamma^{\prime}\right)^{+}$. By the ellipticity condition Assumption 5.1(iii) and the Lipschitz continuity condition in Assumption 5.1(iv) we then have

$$
G(\theta, r, \beta, \gamma)=G\left(\theta, r, \beta, \gamma-\gamma^{\prime}+\gamma^{\prime}\right) \leq G\left(\theta, r, \beta,\left(\gamma-\gamma^{\prime}\right)^{+}+\gamma^{\prime}\right) \leq L\left(\gamma-\gamma^{\prime}\right)+G\left(\theta, r, \beta, \gamma^{\prime}\right),
$$

which concludes the proof.

Lemma 5.6. Recall the definition of the nonlinear expectation $\underline{\mathscr{E}}_{L}^{\prime}$ in Remark 3.2. Let $\xi: \Omega \rightarrow \mathbb{R}$ be bounded, $|\cdot|_{\infty}$-u.s.c. Define

$$
\phi(\theta):=\underline{\mathscr{E}}_{L}^{\prime}\left[\xi^{\theta}\right]+\varphi^{\alpha, \beta, \gamma}(\theta) .
$$

Then $\phi$ is a locally bounded $d_{\infty}$-u.s.c. function and is a $\mathscr{P}_{L}$-viscosity subsolution to

$$
-\partial_{t} \phi+\alpha+L\left|\beta+\gamma \omega_{t}-\partial_{\omega} \phi\right|+L\left(\gamma-\partial_{\omega \omega}^{2} \phi\right)^{+}=0 .
$$

Proof. It is clear that $\phi$ is a locally bounded $d_{\infty}$-u.s.c. function. Define

$$
\psi(\theta):=\phi(\theta)-\varphi^{\alpha, \beta, \gamma}(\theta)=\underline{\mathscr{E}}_{L}^{\prime}\left[\xi^{\theta}\right] .
$$

By the dynamic programming result (see e.g. Theorem 2.3 in [18]), we have

$$
\psi(\theta)=\underline{\mathscr{E}}^{\prime} L\left[\psi^{\theta}(\eta, \mathrm{B})\right] \text { for all } \mathbb{F} \text {-stopping time } \eta \geq 0 .
$$

Then it is easy to verify $\psi$ is a $\mathscr{P}_{L}$-viscosity subsolution to

$$
-\partial_{t} \psi+L\left|\partial_{\omega} \psi\right|+L\left(-\partial_{\omega \omega}^{2} \psi\right)^{+}=0 .
$$

and, using (5.3), it is not difficult too see that $\phi$ is a $\mathscr{P}_{L}$-viscosity subsolution to (5.2).

Lemma 5.7. Let $\xi: \Omega \rightarrow \mathbb{R}$ be bounded and $d_{\infty}$-l.s.c. Define

$$
\phi(\theta):=\underline{\mathscr{E}}_{L}^{\prime}\left[\xi^{\theta}\right]
$$

Then $\phi$ is a bounded $d_{\infty}$-l.s.c. function.

Proof. Define the function

$$
f(\theta, \mathbb{P}):=\mathbb{E}^{\mathbb{P}}\left[\xi^{\theta}\right], \quad \text { for all } \theta \in \Theta \text { and } \mathbb{P} \in \mathscr{P}_{L}^{\prime} .
$$

Let $\theta_{n} \in \Theta$ and $\mathbb{P}_{n} \in \mathscr{P}_{L}^{\prime}$ such that $d_{\infty}\left(\theta_{n}, \theta\right) \rightarrow 0$ and $\mathbb{P}_{n} \rightarrow \mathbb{P}$. By Skorohod's representation there exists a probability space $(\widehat{\Omega}, \widehat{\mathscr{G}}, \widehat{\mathbb{P}})$ on which

$$
\exists \text { r.v.'s }\left.\left.\quad \mathrm{X}^{n}\right|_{\widehat{\mathbb{P}}} \stackrel{d}{=} \mathrm{X}\right|_{\mathbb{P}_{n}},\left.\left.\quad \mathrm{X}^{*}\right|_{\widehat{\mathbb{P}}} \stackrel{d}{=} \mathrm{X}\right|_{\mathbb{P}} \text { such that }\left|\mathrm{X}^{n}-\mathrm{X}^{*}\right|_{\infty} \rightarrow 0, \quad \widehat{\mathbb{P}} \text {-a.s. }
$$

So we have

$$
\liminf _{n \rightarrow \infty} f\left(\theta_{n}, \mathbb{P}_{n}\right)=\liminf _{n \rightarrow \infty} \mathbb{\mathbb { P }}^{\widehat{P}}\left[\xi\left(\omega_{n} \otimes_{t_{n}} \mathrm{~B}^{n}\right)\right] \geq \mathbb{E}^{\widehat{P}}\left[\xi\left(\omega \otimes_{t} \mathrm{~B}^{*}\right)\right]=f(\theta, \mathbb{P}),
$$

where the inequality is due to Fatou's lemma and the $d_{\infty}$-l.s.c. of $\xi$. Therefore, $f$ is l.s.c. on $\Theta \times \mathscr{P}_{L}^{\prime}$. Further, note that

$$
\phi(\theta)=\sup _{\mathbb{P} \in \mathscr{P}_{L}^{\prime}} f(\theta, \mathbb{P})
$$

and $\mathscr{P}_{L}^{\prime}$ is compact (see [17]). By Proposition 7.32 of [1], we obtain that $\phi$ is $d_{\infty}$-l.s.c. 
Lemma 5.8. Let Assumption 5.1 hold true. Let $u$ be a bounded $\mathscr{P}_{L}$-viscosity subsolution (resp. supersolution) and $u^{*}$ (resp. $u_{*}$ ) be its $d$-u.s.c. (resp. l.s.c.) envelop. Then $u^{*}$ (resp. $u_{*}$ ) is also a $\mathscr{P}_{L}$-viscosity subsolution (resp. supersolution).

Proof. We only show the result for subsolution. The one for the supersolution follows from the same argument. First, notice that $d \ll d_{\infty}$ implies that $u^{*}$ is $d_{\infty}$-u.s.c. Now let $\theta \in \Theta$ and $(\alpha, \beta, \gamma) \in$ $\mathscr{J} u^{*}(\theta)$. By the very definition of $u^{*}$, it is clear that the assumptions of Proposition 5.4 are fulfilled (with the functions $u_{n}, \bar{u}$ appearing in the statement replaced by $u, u^{*}$, respectively). Hence there exist $\hat{\theta}_{n} \in \Theta,\left(\alpha_{n}, \beta_{n}, \gamma\right) \in \mathscr{J} u\left(\hat{\theta}_{n}\right)$ such that

$$
d\left(\hat{\theta}_{n}, \theta\right) \rightarrow 0, \quad\left(u\left(\hat{\theta}_{n}\right), \alpha_{n}, \beta_{n}\right) \rightarrow\left(u^{*}(\theta), \alpha, \beta\right) .
$$

Since $u$ is a $\mathscr{P}_{L}$-viscosity subsolution to (3.1), we have

$$
-\alpha_{n}-G\left(\hat{\theta}_{n}, u\left(\hat{\theta}_{n}\right), \beta_{n}, \gamma\right) \leq 0 .
$$

By letting $n \rightarrow \infty$, we have $-\alpha-G\left(\theta, u^{*}(\theta), \beta, \gamma\right) \leq 0$.

Lemma 5.9. For $\theta \in \Theta$, with $t<T$, define

$$
\begin{aligned}
\mathscr{D}(\theta):=\{\phi:[0, T-t] \times \Omega \rightarrow \mathbb{R}: & \underline{u}^{\theta} \leq \phi \leq \bar{v}^{\theta} \text { and } \\
& \phi \text { is a bounded d-u.s.c. viscosity subsolution of (3.1) on }[0, T-t]\},
\end{aligned}
$$

and $\widetilde{u}\left(\theta^{\prime}\right):=\sup \left\{\phi\left(\theta^{\prime}\right): \phi \in \mathscr{D}(\theta)\right\}$. Then we have $u(\theta)=\widetilde{u}(0)$.

Proof. First it is obvious that, for any $\phi \in \mathscr{D}$, we have $\phi^{\theta} \in \mathscr{D}(\theta)$. So $u(\theta) \leq \widetilde{u}(0)$.

On the other hand, suppose $u(\theta)<\widetilde{u}(0)$. Then there is $\phi \in \mathscr{D}(\theta)$ such that $\phi(0)>u(\theta)$. Now take any $\widetilde{\phi} \in \mathscr{D}$, and define, for $\theta^{\prime} \in \Theta$,

$$
\Phi\left(\theta^{\prime}\right):= \begin{cases}\widetilde{\phi}\left(\theta^{\prime}\right) \vee \phi(\hat{\theta}) & \text { if } \hat{t}+t=t^{\prime} \geq t \text { and } \omega^{\prime}=\omega \otimes_{t} \hat{\omega} \\ \widetilde{\phi}\left(\theta^{\prime}\right) & \text { elsewhere. }\end{cases}
$$

It is easy to verify that $\Phi$ is $d_{\infty}$-u.s.c. and that, by the viscosity subsolution property of $\widetilde{\phi}$ and $\phi$, $\Phi$ is a $\mathscr{P}_{L}$-viscosity subsolution to (3.1). Then, it follows from Lemma 5.8 that the $d$-u.s.c. envelop $\Phi^{*}$ is also a $\mathscr{P}_{L}$-viscosity subsolution to (3.1). Finally note that

$$
\Phi^{*}(\theta) \geq \Phi(\theta) \geq \phi(0)>u(\theta)
$$

which is a contradiction to the definition of $u$. Therefore, we have $u(\theta)=\widetilde{u}(0)$.

Proof of Theorem 5.3. Thanks to Lemma 5.9, we only need to check the property of viscosity solution at the point $\theta=(0,0)$.

Step 1. We first prove that $u$ is $d$-u.s.c. and that it is a $\mathscr{P}_{L}$-viscosity subsolution. Let $(\alpha, \beta, \gamma) \in$ $\overline{\mathscr{J}} u^{*}(0,0)$, where $u^{*}$ is the $d$-u.s.c. envelop of $u$. By the definition of $u$ and $u^{*}$, there exists a sequence of $u_{n} \in \mathscr{D}$ and $\theta_{n} \in \Theta$ such that

$$
d\left(\theta_{n},(0,0)\right) \rightarrow 0, u_{n}\left(\theta_{n}\right) \rightarrow u^{*}(0,0) .
$$

Moreover,

$$
\text { if } \theta_{n}^{\prime} \in \Theta \text { and } d\left(\theta_{n}^{\prime}, \theta^{\prime}\right) \rightarrow 0 \text {, then } \limsup _{n \rightarrow \infty} u_{n}\left(\theta_{n}^{\prime}\right) \leq u^{*}\left(\theta^{\prime}\right) \text {. }
$$


It follows from Proposition 5.4 that there exist $\hat{\theta}_{n} \in \Theta$ and $\left(\alpha_{n}, \beta_{n}, \gamma\right) \in \mathscr{J} u_{n}\left(\hat{\theta}_{n}\right)$ such that

$$
d\left(\hat{\theta}_{n},(0,0)\right) \rightarrow 0, \quad\left(u_{n}\left(\hat{\theta}_{n}\right), \alpha_{n}, \beta_{n}\right) \rightarrow\left(u^{*}(0,0), \alpha, \beta\right) .
$$

Since $u_{n}$ are all $\mathscr{P}_{L}$-viscosity subsolution to (3.1), we have

$$
-\alpha_{n}-G\left(\hat{\theta}_{n}, u_{n}\left(\hat{\theta}_{n}\right), \beta_{n}, \gamma\right) \leq 0 .
$$

If we let $n \rightarrow \infty$, we obtain $-\alpha-G\left((0,0), u^{*}(0,0), \beta, \gamma\right) \leq 0$. Therefore, $u^{*}$ is a $\mathscr{P}_{L}$-viscosity subsolution. By definition of $u$, we have $u^{*} \leq u$. On the other hand, by definition of $u^{*}$, we also have $u^{*} \geq u$. So finally we conclude that

$$
u=u^{*}
$$

is $d$-u.s.c. and a $\mathscr{P}_{L}$-viscosity subsolution.

Step 2. Let $\widetilde{u}_{*}$ be the $d_{\infty}$-l.s.c. envelop of the function $u$. We now prove $\widetilde{u}_{*}$ is a $\mathscr{P}_{L}$-viscosity supersolution at $\theta=0$. Suppose it is not the case and there is $(\alpha, \beta, \gamma) \in \overline{\mathscr{J}} \widetilde{u}_{*}(0,0)$ such that

$$
-\alpha-G\left((0,0), \widetilde{u}_{*}(0,0), \beta, \gamma\right)=-3 \varepsilon<0 .
$$

Therefore, for $\delta$ small enough we have

$$
\begin{gathered}
\widetilde{u}_{*}(0,0)=\underline{\mathscr{E}}_{L}\left[\left(\widetilde{u}_{*}-\varphi^{\alpha, \beta, \gamma}\right)\left(\mathrm{T} \wedge \mathrm{H}_{\delta}, \mathrm{B}\right)\right], \\
\delta \leq \frac{\varepsilon}{L|\gamma|} \quad \text { and } \quad-\alpha-G\left(\theta, \widetilde{u}_{*}(\theta), \beta+\gamma \omega_{t}, \gamma\right)<-2 \varepsilon \quad \text { for all } t \leq \mathrm{H}_{\delta}(\omega) .
\end{gathered}
$$

For the simplicity of notation, we denote for all $\theta \in \Theta$

$$
\xi(\theta):=u\left(\mathrm{H}_{\delta}(\omega), \omega\right)-\varphi^{\alpha-\varepsilon, \beta, \gamma}\left(\mathrm{H}_{\delta}(\omega), \omega\right) \quad \text { and } \quad \underline{\xi}(\theta):=\widetilde{u}_{*}\left(\mathrm{H}_{\delta}(\omega), \omega\right)-\varphi^{\alpha-\varepsilon, \beta, \gamma}\left(\mathrm{H}_{\delta}(\omega), \omega\right) .
$$

Recall the nonlinear expectation $\underline{\mathscr{E}}_{L}^{\prime}$ defined in Remark 3.2. Define

$$
\phi(\theta):=\underline{\mathscr{E}}_{L}^{\prime}\left[\xi^{\theta}\right]+\varphi^{\alpha-\varepsilon, \beta, \gamma}(\theta), \quad \underline{\phi}(\theta):=\underline{\mathscr{E}}_{L}^{\prime}\left[\underline{\xi}^{\theta}\right]+\varphi^{\alpha-\varepsilon, \beta, \gamma}(\theta) .
$$

By recalling the definitions of $\underline{\mathscr{E}}_{L}^{\prime}$ and $\underline{\mathscr{E}}_{L}$, we have

$$
\underline{\phi}(0,0)=\underline{\mathscr{E}}_{L}^{\prime}[\underline{\xi}] \geq \underline{\mathscr{E}}_{L}[\underline{\xi}]=\underline{\mathscr{E}}_{L}\left[\left(\widetilde{u}_{*}-\varphi^{\alpha-\varepsilon, \beta, \gamma}\right)\left(\mathrm{H}_{\delta}, \mathrm{B}\right)\right]>\widetilde{u}_{*}(0,0) .
$$

By Lemma 5.7, $\phi$ is $d_{\infty}$-l.s.c. By Step 1 we know that $u$ is $d$ - hence $d_{\infty}$-u.s.c. Then, by Lemma 5.6, it follows that $\bar{\phi}$ is a locally bounded $d_{\infty}$-u.s.c. function and a $\mathscr{P}_{L}$-viscosity subsolution to

$$
-\partial_{t} \phi+\alpha-\varepsilon+L\left|\beta+\gamma \omega_{t}-\partial_{\omega} \phi\right|+L\left(\gamma-\partial_{\omega \omega}^{2} \phi\right)^{+}=0 .
$$

It follows from (5.6) that $\alpha>2 \varepsilon-G\left(\theta, \widetilde{u}_{*}(\theta), \beta+\gamma \omega_{t}, \gamma\right)$ on $\left\{\theta: t<\mathrm{H}_{\delta}(\omega)\right\}$. By the Lipschitz continuity assumptions on $G$ and Lemma 5.5 , we then have that $\phi$ is a $\mathscr{P}_{L}$-viscosity subsolution to

$$
-\partial_{t} \phi-G\left(\cdot, \widetilde{u}_{*}, \partial_{\omega} \phi, \partial_{\omega \omega}^{2} \phi\right)=0, \quad \text { on } \quad\left\{\theta: t<\mathrm{H}_{\delta}(\omega)\right\} .
$$

Further, since $\widetilde{u}_{*} \leq u \leq u \vee \phi$ and $G$ is non-decreasing in $r$, the function $\phi$ is a $\mathscr{P}_{L}$-viscosity subsolution to

$$
-\partial_{t} \phi-G\left(\cdot, u \vee \phi, \partial_{\omega} \phi, \partial_{\omega \omega}^{2} \phi\right)=0, \quad \text { on } \quad\left\{\theta: t<\mathrm{H}_{\delta}(\omega)\right\} .
$$

Now define

$$
U:=(u \vee \phi) 1_{\left\{t<\mathrm{H}_{\delta}(\omega)\right\}}+u 1_{\left\{t \geq \mathrm{H}_{\delta}(\omega)\right\}} .
$$


Recall that $u, \phi$ are both $d_{\infty}$-u.s.c. and that $\mathrm{H}_{\delta}$ is continuous, and observe that $u=\phi$ on $\left\{t=\mathrm{H}_{\delta}(\omega)\right\}$. So $U$ is $d_{\infty}$-u.s.c. Further, since $u$ is a $\mathscr{P}_{L}$-viscosity subsolution to (3.1) and $\phi$ is a $\mathscr{P}_{L}$-viscosity subsolution to (5.8) on $\left\{t<\mathrm{H}_{\delta}(\omega)\right\}$, it is easy to verify that $U$ is a $\mathscr{P}_{L}$-viscosity subsolution to (3.1). Then it follows from Lemma 5.8 that the $d$-u.s.c. envelop of $U$, namely $U^{*}$, is a $\mathscr{P}_{L}$-viscosity subsolution to (3.1).

However, by definition of $\widetilde{u}_{*}$ there is a sequence $\theta_{n} \in \Theta$ such that $d_{\infty}\left(\theta_{n},(0,0)\right) \rightarrow 0$ and $\widetilde{u}_{*}(0,0)=\lim _{n \rightarrow \infty} u\left(\theta_{n}\right)$, and thus

$$
\liminf _{n \rightarrow \infty}\left(U^{*}\left(\theta_{n}\right)-u\left(\theta_{n}\right)\right) \geq \liminf _{n \rightarrow \infty}\left(\phi\left(\theta_{n}\right)-u\left(\theta_{n}\right)\right) \geq \liminf _{n \rightarrow \infty}\left(\underline{\phi}\left(\theta_{n}\right)-u\left(\theta_{n}\right)\right) \geq \underline{\phi}(0,0)-\widetilde{u}_{*}(0,0)>0 .
$$

The second last inequality is due to the $d_{\infty}$-l.s.c. of the function $\phi$. Therefore, there is $\theta_{n}$ such that $U^{*}\left(\theta_{n}\right)>u\left(\theta_{n}\right)$, which is in contradiction with the definition of $u$.

Step 3. By Step 2 and Lemma 5.8, the $d$-l.s.c. envelop of $\widetilde{u}_{*}$, namely $\left(\widetilde{u}_{*}\right)_{*}$, is a $\mathscr{P}_{L}$-viscosity supersolution to (3.1). Since $\widetilde{u}_{*} \leq u$, we have $\left(\widetilde{u}_{*}\right)_{*} \leq u_{*}$. On the other hand, since $\widetilde{u}_{*} \geq u_{*}$, we have $\left(\widetilde{u}_{*}\right)_{*} \geq u_{*}$. Therefore, $u_{*}=\left(\widetilde{u}_{*}\right)_{*}$ is a $\mathscr{P}_{L}$-viscosity supersolution to (3.1).

Step 4. Note that $\underline{u}_{*} \leq u_{*} \leq \bar{v}$, in particular, $u_{*}(T, \cdot)=\xi$. By Step 3 and Assumption 5.2, we $\overline{\text { have } u} \leq u_{*}$. Together with the definition of $u_{*}$, we have that $u=u_{*}$ is $d$-l.s.c. and a $\mathscr{P}_{L}$-viscosity supersolution to (3.1). Then, recalling what proved in Step 1, we conclude that $u$ is a $d$-continuous $\mathscr{P}_{L}$-viscosity solution to (3.1).

\section{Comparison result for $\overleftarrow{d}_{p}$-semicontinuous solutions}

We have seen that for Perron's method the comparison result for semicontinuous solutions is crucial. However, up to now there is no such result for the fully nonlinear path-dependent PDE in the literature. The main difficulty, as explained in Remark 3.2, is due to the optimal stopping problem presented in (3.4). As an advantage of our modified definition of $\mathscr{P}_{L}$-viscosity solutions, we are able to show such a comparison result by combining the comparison result for uniformly continuous solutions proved in [28] and the convolution in backward pseudo-metric developed in [24].

In the present section we will deal with the pseudo-metric $\overleftarrow{d}_{p}$ on $\Theta$ defined, for $1 \leq p<\infty$, by

$$
\overleftarrow{d}_{p}\left(\theta, \theta^{\prime}\right):=\left|t-t^{\prime}\right|+\left|\omega_{t}-\omega_{t^{\prime}}^{\prime}\right|+\left(\int_{0}^{T}\left|\omega_{t-s}-\omega_{t^{\prime}-s}^{\prime}\right|^{p} d s\right)^{\frac{1}{p}} \quad \forall \theta, \theta^{\prime} \in \Theta
$$

where we extend $\omega, \omega^{\prime}$ on the negative real line by $\omega_{s}=\omega_{s}^{\prime}=0$ for $s<0$. Notice that $\overleftarrow{d}_{p} \ll d_{\infty}$.

Remark 6.1. Let us we recall the pseudo-metric $d_{p}(1 \leq p<\infty)$ on $\Theta$ defined in [28]:

$$
d_{p}\left(\theta, \theta^{\prime}\right):=\left|t-t^{\prime}\right|+\left(\int_{0}^{T+1}\left|\omega_{t \wedge s}-\omega_{t^{\prime} \wedge s}^{\prime}\right|^{p} d s\right)^{\frac{1}{p}} \quad \forall \theta, \theta^{\prime} \in \Theta
$$

where we set $\omega_{s}:=\omega_{t}, \omega_{s}^{\prime}:=\omega_{t^{\prime}}^{\prime}$ for $s \in[T, T+1]$. It is not difficult to see that the two pseudometrics $\overleftarrow{d}_{p}, d_{p}$ induce the same topology on $\Theta$, i.e., the identity map $\left(\Theta, \overleftarrow{d}_{p}\right) \rightarrow\left(\Theta, d_{p}\right)$ is a homeomorphism. In particular, the classes of upper-/lower-semicontinous functions with respect to $\overleftarrow{d}_{p}$ and $d_{p}$ coincide. Neverthless, the pseudo-metric uniformities associated with $\overleftarrow{d}_{p}$ and with $d_{p}$ do not coincide, hence uniformly continuous functions with respect to $\overleftarrow{d}_{p}$ may not be uniformly continuous with respect to $d_{p}$, and viceversa.

The comparison result in this section will hold under the following strong assumption on the nonlinearity $G$. 
Assumption 6.2. The function $G: \Theta \times \mathbb{R}^{m} \times \mathbb{S}^{m} \rightarrow \mathbb{R}$ satisfies the following assumptions.

(i) The function $\theta \mapsto G(\theta, \beta, \gamma)$ is $\overleftarrow{d}_{p}$-uniformly continuous, uniformly in $(\beta, \gamma)$

(ii) For every $(\theta, \beta) \in \Theta \times \mathbb{R}^{m}$,

$$
G(\theta, \beta, \gamma) \leq G\left(\theta, \beta, \gamma^{\prime}\right) \quad \forall \gamma, \gamma^{\prime} \in \mathbb{S}^{m}, \gamma \leq \gamma^{\prime} .
$$

(iii) For all $\theta \in \Theta, \beta, \beta^{\prime} \in \mathbb{R}^{m}, \gamma, \gamma^{\prime} \in \mathbb{S}^{m}$,

$$
\left|G\left(\theta, \beta+\beta^{\prime}, \gamma+\gamma^{\prime}\right)-G(\theta, \beta, \gamma)\right| \leq L\left(\left|\beta^{\prime}\right|+\left|\gamma^{\prime}\right|\right) .
$$

Note that if Assumption 6.2 holds true then Assumption 5.1 holds true with $d=\overleftarrow{d}_{p}$.

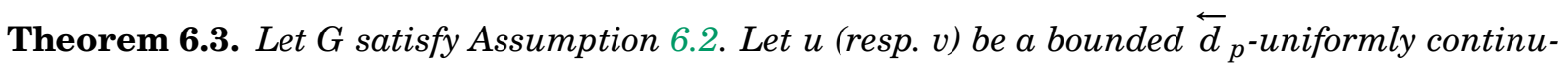
ous $\mathscr{P}_{L}$-viscosity subsolution (resp. supersolution) to PPDE (3.1). If $u(T, \cdot) \leq v(T, \cdot)$, we have $u \leq v$ on $\Theta$.

Proof. A similar comparison result, in which $u, v, G$ are $d_{p}$-uniformly continuous, is proved under the old definition of viscosity solution in [28]. As mentioned in Remark 3.2, the change of the definition does not add trouble for proving the existing comparison result. Further, we can indeed apply the same argument as in [28] to prove the desired comparison result, where the $d_{p}$-uniform continuity is replaced by $\overleftarrow{d}_{p}$-uniform continuity. Since the whole argument is too long, we refer the reader to [28] for the technical details.

In the rest of this section, we show a comparison result for $\overleftarrow{d}_{p}$-semicontinuous solutions. The main idea is to approximate the semicontinuous solutions by uniform continuous functions with the following convolution. For a bounded $\overleftarrow{d}_{p}$-u.s.c. function $u$, we define

$$
u^{n}(\theta):=\sup _{\theta^{\prime} \in \Theta}\left\{u\left(\theta^{\prime}\right)-n \overleftarrow{d}_{p}\left(\theta, \theta^{\prime}\right)\right\}
$$

Then $u^{n}$ is a bounded $\overleftarrow{d}_{p}$-Lipschitz function and $u^{n} \rightarrow u$ pointwise as $n \rightarrow \infty$

Assumption 6.4. For a function $u: \Theta \rightarrow \mathbb{R}$, there is a constant $C_{0}$ such that

$$
\limsup _{\delta \rightarrow 0}\left\{\frac{\left|u\left(\theta^{\prime}\right)-u(T, \omega)\right|}{\overleftarrow{d}_{p}\left(\theta^{\prime},(T, \omega)\right)}: \theta^{\prime} \in \Theta, \omega \in \Omega, \overleftarrow{d}_{p}\left(\theta^{\prime},(T, \omega)\right) \leq \delta\right\} \leq C_{0}
$$

where we adopt the convention $\frac{0}{0}=0$.

Example 6.5. Let $\underline{u}, \bar{v}$ be two $\overleftarrow{d}_{p}$-Lipschitz functions with Lipschitz constant $C$ and assume that $\underline{u}(T, \cdot)=\bar{v}(T, \cdot)$ and $\underline{u} \leq \bar{v}$ on $\Theta$. Then all the functions $u$, such that $\underline{u} \leq u \leq \bar{v}$, satisfy Assumption 6.4 .

Lemma 6.6. Let $u$ be a bounded $\overleftarrow{d}_{p}$-u.s.c. function satisfing Assumption 6.4. Then we have $u^{n}(T, \cdot)=u(T, \cdot)$ with $u^{n}$ defined in (6.1) for $n$ big enough.

Proof. Clearly $u^{n}(T, \cdot) \geq u(T, \cdot)$ for all $n$. Now let $\delta>0$ be small enough such that the supremum appearing in (6.2) is less than $C_{0}+1$. Let $n \geq\left(C_{0}+1\right) \vee \frac{2|u|_{\infty}}{\delta}$. Then

$$
u(T, \omega)=u(T, \omega)-u\left(\theta^{\prime}\right)+u\left(\theta^{\prime}\right) \geq-n \overleftarrow{d}_{p}\left(\theta^{\prime},(T, \omega)\right)+u\left(\theta^{\prime}\right) \quad \forall \theta^{\prime} \in \Theta, \omega \in \Omega
$$

or, equivalently, $u(T, \cdot) \geq u^{n}(T, \cdot)$. 
The main advantage of the convolution in (6.1) is that $u^{n}$ inherits the viscosity subsolution property of $u$. We first prove a lemma, which is an adaptation of the important Lemma 3.3 of [28] to the new definition of $\mathscr{P}_{L}$-viscosity solution. Note that in Lemma 3.3 of [28] the result holds true only for uniformly continuous functions, while the following lemma is proved for u.s.c. functions. This improvement is due to our new definition.

Lemma 6.7. Let $u$ be a $\overleftarrow{d}_{p}$-u.s.c. function satisfying $u(0,0)>\overline{\mathscr{E}}_{L}\left[\left(u-\varphi^{\alpha, \beta, \gamma}\right)\left(\mathrm{H}_{\delta}, \mathrm{B}\right)\right]$, for some $\delta>0$ and $(\alpha, \beta, \gamma) \in \mathbb{R} \times \mathbb{R}^{m} \times \mathbb{S}^{m}$. Then, there exists $\theta^{*} \in \Theta$ such that

$$
t^{*}<\mathrm{H}_{\delta}\left(\omega^{*}\right) \text { and }\left(\alpha, \beta+\gamma \omega_{t^{*}}^{*}, \gamma\right) \in \underline{\mathscr{J}}_{L} u\left(\theta^{*}\right) .
$$

Proof. Let $U:=u-\varphi^{\alpha, \beta, \gamma}$ and define the value function $V: \Theta \rightarrow \mathbb{R}$ by

$$
V(\theta):=\overline{\mathscr{E}}_{L}^{t}\left[U^{\theta}\left(\mathrm{T} \wedge \mathrm{H}_{\delta}, \mathrm{B}\right)\right](\vartheta),
$$

where $\vartheta=(t, \omega, a, \mu, q) \in \widetilde{\Theta}$ is such that $\theta=(t, \omega)$. Notice that the definition of $V(\theta)$ does not depend on the representative $\vartheta$. By compactness of $\mathscr{P}_{L}$ and by upper semicontinuity of the map

$$
\mathscr{P}_{L} \rightarrow \mathbb{R}, \mathbb{P} \mapsto \mathbb{E}^{\mathbb{P}}\left[U\left(\mathrm{~T} \wedge \mathrm{H}_{\delta}, \mathrm{B}\right)\right]
$$

we can find $\mathbb{P}^{*} \in \mathscr{P}_{L}$ such that $V(0,0)=\mathbb{E}^{\mathbb{P}^{*}}\left[U\left(\mathrm{~T} \wedge \mathrm{H}_{\delta}, \mathrm{B}\right)\right]$. By Proposition 4.3 we have

$$
U=V \quad \mathbb{P}^{*} \text {-a.s. }
$$

On the other hand, by the assumption of the lemma, we have

$$
\mathbb{E}^{\mathbb{P}^{*}}\left[U\left(\mathrm{~T} \wedge \mathrm{H}_{\delta}, \mathrm{B}\right)\right]=V(0,0) \geq U(0,0)=u(0,0)>\overline{\mathscr{E}}_{L}\left[U\left(\mathrm{H}_{\delta}, \mathrm{B}\right)\right] \geq \mathbb{E}^{\mathbb{P}^{*}}\left[U\left(\mathrm{H}_{\delta}, \mathrm{B}\right)\right]
$$

Therefore,

$$
\mathbb{P}^{*}\left[\mathrm{~T}<\mathrm{H}_{\delta}\right]>0 .
$$

By taking into account (6.4) and (6.5), we conclude that there exists $\vartheta^{*} \in \widetilde{\Theta}$ such that $t^{*}<\mathrm{H}_{\delta}\left(\omega^{*}\right)$ and $U\left(\theta^{*}\right)=V\left(\theta^{*}\right)$, which is equivalent to (6.3).

If $G$ satisfies Assumption 6.2, we define the modulus of continuity $\rho_{G}$ by

$$
\rho_{G}(x):=\sup _{\beta \in \mathbb{R}^{m}, \gamma \in \mathbb{S}^{m}} \sup _{\substack{\theta, \theta^{\prime} \in \Theta \\ \overleftarrow{d}_{p}\left(\theta, \theta^{\prime}\right) \leq x}}\left|G(\theta, \beta, \gamma)-G\left(\theta^{\prime}, \beta, \gamma\right)\right|, \quad \forall x \geq 0 .
$$

Proposition 6.8. Let u be a $\overleftarrow{d}_{p}$-u.s.c. $\mathscr{P}_{L}$-viscosity subsolution to (3.1), bounded by a constant $C>0$, and let $u^{n}$ be defined as in (6.1). Assume that $G$ satisfies Assumption 6.2. Then, for $n$ big enough, $u^{n}$ is a $\mathscr{P}_{L}$-viscosity subsolution to the following equation:

$$
-\partial_{t} u^{n}-G\left(\theta, \partial_{\omega} u^{n}, \partial_{\omega \omega}^{2} u^{n}\right) \leq \rho_{G}\left(\frac{2 C+1}{n}\right) .
$$

Proof. Let $(\alpha, \beta, \gamma) \in \underline{\mathscr{J}}_{L} u^{n}(\theta)$. Then for any $\varepsilon>0$ we have

$$
u^{n}(\theta)>\overline{\mathscr{E}}_{L}\left[\left(\left(u^{n}\right)^{\theta}-\varphi^{\alpha+\varepsilon, \beta, \gamma}\right)\left(\mathrm{H}_{\delta}, \mathrm{B}\right)\right],
$$

for a suitably $\delta>0$ arbitrarily small. By definition of $u^{n}$ and by (6.6), we can find $\theta^{*} \in \Theta$ such that

$$
\overleftarrow{d}_{p}\left(\theta, \theta^{*}\right)<\frac{2 C+1}{n} \text { and } u\left(\theta^{*}\right)-n \overleftarrow{d}_{p}\left(\theta, \theta^{*}\right)>\overline{\mathscr{E}}_{L}\left[\left(\left(u^{n}\right)^{\theta}-\varphi^{\alpha+\varepsilon, \beta, \gamma}\right)\left(\mathrm{H}_{\delta}, \mathrm{B}\right)\right]
$$


Further, we have

$$
\begin{aligned}
u\left(\theta^{*}\right)-n \overleftarrow{d}_{p}\left(\theta, \theta^{*}\right) & >\overline{\mathscr{E}}_{L}\left[\left(\left(u^{n}\right)^{\theta}-\varphi^{\alpha+\varepsilon, \beta, \gamma}\right)\left(\mathrm{H}_{\delta}, \mathrm{B}\right)\right] \\
& \geq \overline{\mathscr{E}}_{L}\left[\left(u^{\theta^{*}}-\varphi^{\alpha+\varepsilon, \beta, \gamma}\right)\left(\mathrm{H}_{\delta}, \mathrm{B}\right)-n \overleftarrow{d}_{p}\left(\left(t+\mathrm{H}_{\delta}, \omega \otimes_{t} \mathrm{~B}\right),\left(t^{*}+\mathrm{H}_{\delta}, \omega^{*} \otimes_{t^{*}} \mathrm{~B}\right)\right)\right]
\end{aligned}
$$

It is important to note that

$$
\overleftarrow{d}_{p}\left(\theta, \theta^{*}\right)=\overleftarrow{d}_{p}\left(\left(t+\mathrm{H}_{\delta}, \omega \otimes_{t} \mathrm{~B}\right),\left(t^{*}+\mathrm{H}_{\delta}, \omega^{*} \otimes_{t^{*}} \mathrm{~B}\right)\right)
$$

Therefore,

$$
u\left(\theta^{*}\right)>\overline{\mathscr{E}}_{L}\left[\left(u^{\theta^{*}}-\varphi^{\alpha+\varepsilon, \beta, \gamma}\right)\left(\mathrm{H}_{\delta}, \mathrm{B}\right)\right] .
$$

Now we apply Lemma 6.7 and obtain that there exists $\widetilde{\theta}$ such that

$$
\widetilde{t}<\mathrm{H}_{\delta}(\widetilde{\omega}) \quad \text { and } \quad\left(\alpha+\varepsilon, \beta+\gamma \widetilde{\omega}_{\tilde{t}}, \gamma\right) \in \underline{J}_{L} u\left(t^{*}+\widetilde{t}, \omega^{*} \otimes_{t^{*}} \widetilde{\omega}\right),
$$

and thus, by the subsolution property of $u$,

$$
-\alpha-\varepsilon-G\left(t^{*}+\widetilde{t}, \omega^{*} \otimes_{t^{*}} \widetilde{\omega}, \beta+\gamma \widetilde{\omega}_{\widetilde{t}}, \gamma\right) \leq 0 .
$$

Note that

$$
\begin{aligned}
\overleftarrow{d}_{p}\left(\theta,\left(t^{*}+\widetilde{t}, \omega^{*} \otimes_{t^{*}} \widetilde{\omega}\right)\right) & \leq \overleftarrow{d}_{p}\left(\left(t+\widetilde{t}, \omega \otimes_{t} \widetilde{\omega}\right),\left(t^{*}+\widetilde{t}, \omega^{*} \otimes_{t^{*}} \widetilde{\omega}\right)\right)+\overleftarrow{d}_{p}\left(\theta,\left(t+\widetilde{t}, \omega \otimes_{t} \widetilde{\omega}\right)\right) \\
& \leq \overleftarrow{d}_{p}\left(\theta, \theta^{*}\right)+\delta+\left(\delta^{p+1}+T\left|\rho\left(\omega_{t \wedge \cdot}, \delta\right)\right|^{p}\right)^{\frac{1}{p}} \\
& \leq \frac{2 C+1}{n}+\delta+\left(\delta^{p+1}+T\left|\rho\left(\omega_{t \wedge .}, \delta\right)\right|^{p}\right)^{\frac{1}{p}}
\end{aligned}
$$

where $\rho\left(\omega_{t \wedge .}, \cdot\right)$ is the modulus of continuity of the path $\omega_{t \wedge}$. . By using the definition of $\rho_{G}$ together with (6.7) and (6.8), we get

$$
-\alpha-\varepsilon-G\left(\theta, \beta+\gamma \widetilde{\omega}_{\tilde{t}}, \gamma\right) \leq \rho_{G}\left(\frac{2 C+1}{n}+\delta+\left(\delta^{p+1}+T\left|\rho\left(\omega_{t \wedge .}, \delta\right)\right|^{p}\right)^{\frac{1}{p}}\right) .
$$

Finally, we let $\delta, \varepsilon$ tend to 0 and obtain $-\alpha-G(\theta, \beta, \gamma) \leq \rho_{G}\left(\frac{2 C+1}{n}\right)$.

Now we are ready to prove the main result of this section.

Theorem 6.9. Let $G$ satisfy Assumption 6.2. Let u (resp. v) be a bounded $\overleftarrow{d}_{p}$-u.s.c. (resp. l.s.c.) $\mathscr{P}_{L}$-viscosity subsolution (resp. supersolution) to PPDE (3.1). In addition, assume that u,v satisfy Assumption 6.4. Then, if $u(T, \cdot) \leq v(T, \cdot)$, we have $u \leq v$ on $\Theta$.

Proof. As a direct consequence of Proposition 6.8, we know that, for $n$ sufficiently large,

$$
\widetilde{u}^{n}(\theta):=u^{n}(\theta)-\rho_{G}\left(\frac{2 C+1}{n}\right)(T-t)
$$

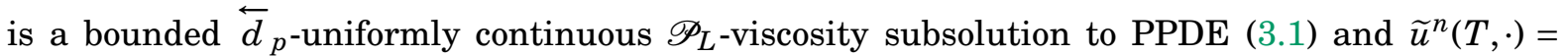
$u^{n}(T, \cdot)$. Further, by Lemma 6.6 , we have $u^{n}(T, \cdot)=u(T, \cdot)$ and thus $\widetilde{u}^{n}(T, \cdot)=u(T, \cdot)$ for $n$ big enough. We can similarly define $\widetilde{v}^{n}$, so that $\widetilde{v}^{n} \rightarrow v$ pointwise and that $\widetilde{v}^{n}$ is a bounded $\overleftarrow{d}_{p^{-}}$ uniformly continuous $\mathscr{P}_{L}$-viscosity supersolution to (3.1) and $\widetilde{v}^{n}(T, \cdot)=v(T, \cdot)$ for $n$ big enough. Since $\widetilde{v}^{n}(T, \cdot)=v(T, \cdot) \geq u(T, \cdot)=\widetilde{u}^{n}(T, \cdot)$, by Theorem 6.3 we have

$$
u=\lim _{n \rightarrow \infty} \widetilde{u}^{n} \leq \lim _{n \rightarrow \infty} \widetilde{v}^{n}=v,
$$

and the proof is complete. 


\section{Representation of PPDEs as PDEs in infinite dimension and comparison under weak-continuity}

The aim of this section is to start with a $\mathscr{P}_{L}$-viscosity (sub-/super-)solution $u$ defined on the space $\Theta$, then to associate to it a function $\widetilde{u}$ defined on a product space $[0, T] \times H$ where $H$ is a suitably chosen Hilbert space, and finally show that $\widetilde{u}$ is a viscosity (sub-/super-)solution of a PDE on $[0, T] \times H$. As a corollary of such relationship, we can exploit the comparison theorem available for viscosity solutions in Hilbert spaces to obtain uniqueness for $\mathscr{P}_{L}$-viscosity solutions.

We start by introducing the Hilbert space $H$ on which we will set our new PDE associated with the original PPDE (3.1). Then we will address the problem of associating a function on the original space $\Theta$ with a function on the product space $[0, T] \times H$. In order to perform this change of variable in such a way to end up with a function regular enough to exploit the comparison theorem for viscosity solutions in Hilbert spaces, we need to introduce a pseudo-metric $d_{B}$ on $\Theta$, weaker than $\overleftarrow{d}_{p}$, and an associated norm $|\cdot|_{B}$ on $H$, weaker than the original norm $|\cdot|_{H}$

Once provided these preliminaries, we can introduce the PDE on $[0, T] \times H$ associated with the original PPDE on $\Theta$, recall the notion of viscosity solution in Hilbert spaces, and prove the main theorem of this section (Theorem 7.6), thanks to which we can use [12, Theorem 3.50] to get uniqueness of $\mathscr{P}_{L}$-viscosity solutions (Corollary 7.7).

For the theory of viscosity solutions in Hilbert spaces we always refer to [12]. For the basic notions of stochastic calculus in Hilbert spaces that we need, we refer to [21].

We start by introducing the Hilbert spaces

$$
H^{\prime}:=L^{2}\left(\mathbb{R}_{-}, \mathbb{R}^{m}\right), \quad H:=\mathbb{R}^{m} \times H^{\prime},
$$

where $H^{\prime}$ is endowed with its standard scalar product $\langle\cdot, \cdot\rangle_{L^{2}}$ induced by the $L^{2}$-norm and $H$ is endowed with scalar product and norm given by

$$
\left\langle x, x^{\prime}\right\rangle_{H}:=\left\langle x_{0}, x_{0}^{\prime}\right\rangle+\left\langle x_{1}, x_{1}^{\prime}\right\rangle_{L^{2}}, \quad|x|_{H}:=\left(\left|x_{0}\right|^{2}+\left|x_{1}\right|_{L^{2}}^{2}\right)^{1 / 2}, \quad \forall x=\left(x_{0}, x_{1}\right), x^{\prime}=\left(x_{0}^{\prime}, x_{1}^{\prime}\right) \in \mathbb{R}^{m} \times H^{\prime} .
$$

We next consider the $C_{0}$-semigroup $S$ on $H$ defined by

$$
S_{t}: H \rightarrow H,\left(x_{0}, x_{1}\right) \mapsto\left(x_{0}, x_{0} \mathbf{1}_{[-t, 0]}+x_{1}(\cdot+t) \mathbf{1}_{(-\infty,-t)}\right) \quad \forall t \in \mathbb{R}^{+} .
$$

The infinitesimal generator $A$ of $S$ is given by

$$
A: D(A) \subset H \rightarrow H,\left(x_{0}, x_{1}\right) \mapsto\left(0, \dot{x}_{1}\right)
$$

where

$$
D(A):=\left\{\left(x_{0}, x_{1}\right) \in H: x_{1} \in W^{1,2}\left(\mathbb{R}^{-}, \mathbb{R}\right), x_{0}=x_{1}(0)\right\} .
$$

To express the regularity assumptions for the comparison results (Theorem 7.6 and Corollary 7.7), we will consider the following pseudo-metric $d_{B}$ on $\Theta$ :

$$
\begin{aligned}
\left.d_{B}\left(\theta, \theta^{\prime}\right)\right):= & \left|t-t^{\prime}\right|+\left|\omega(t)-\omega^{\prime}\left(t^{\prime}\right)\right|+\left|\int_{0}^{t} \omega(r) d r-\int_{0}^{t^{\prime}} \omega^{\prime}(r) d r\right| \\
& +\left(\int_{0}^{T}\left(\int_{(t-\rho) \vee 0}^{t} \omega(r) d r-\int_{\left(t^{\prime}-\rho\right) \vee 0}^{t^{\prime}} \omega^{\prime}(r) d r\right)^{2} d \rho\right)^{1 / 2}
\end{aligned}
$$

for all $\theta, \theta^{\prime} \in \Theta$, and the following scalar product $\langle\cdot, \cdot\rangle_{B}$ and norm $|\cdot|_{B}$ on $H$ :

$$
\left\langle x, x^{\prime}\right\rangle_{B}:=\left\langle(A-I)^{-1} x,(A-I)^{-1} x^{\prime}\right\rangle_{H} \quad|x|_{B}:=\left|(A-I)^{-1} x\right|_{H} \quad \forall x, x^{\prime} \in H .
$$


By a direct computation, one can verify that

$$
(A-I)^{-1}\left(x_{0}, x_{1}\right)=\left(-x_{0},-e \cdot x_{0}-\int^{0} e^{-(r-\cdot)} x_{1}(r) d r\right) \quad \forall x=\left(x_{0}, x_{1}\right) \in H .
$$

Hence the norm $|\cdot|_{B}$ reads

$$
|x|_{B}=\left(\left|x_{0}\right|^{2}+\int_{-\infty}^{0}\left|e^{s} x_{0}+\int_{s}^{0} e^{s-r} x_{1}(r) d r\right|^{2} d s\right)^{1 / 2} \quad \forall x \in H .
$$

By the very definition of $d_{B}$ and $|\cdot|_{B}$, we immediately have the following

Lemma 7.1. Let $\left\{\theta_{n}\right\}_{n} \subset \Theta$ be a sequence and let $\theta \in \Theta$. Define $x_{0}^{n}:=\omega_{n}\left(t_{n}\right), x_{1}^{n}:=\omega_{n}\left(\cdot+t_{n}\right) \mathbf{1}_{\left[-t_{n}, 0\right]}$, $x_{0}:=\omega(t), x_{1}:=\omega(\cdot+t) \mathbf{1}_{[-t, 0]}$. Then (with $x^{n}=\left(x_{0}^{n}, x_{1}^{n}\right), x=\left(x_{0}, x_{1}\right)$ )

$$
t_{n} \rightarrow t \text { and }\left|x^{n}-x\right|_{B} \rightarrow 0 \text { if and only if } d_{B}\left(\theta_{n}, \theta\right) \rightarrow 0 .
$$

The following proposition provides an example of a functional on $\Theta$ which is $d_{B}$-continuous. The proof is postponed to Appendix.

Proposition 7.2. Let $f \in L^{2}\left((0, T), \mathbb{R}^{m}\right)$. Then the convolution

$$
\Theta \rightarrow \mathbb{R}, \theta \mapsto \int_{0}^{t}\langle f(r), \omega(t-r)\rangle d r
$$

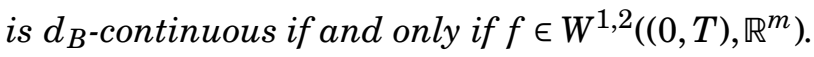

We now define the data for the PDE on $[0, T] \times H$ associated with the original PPDE (3.1). Let the functions

$$
G^{*_{H}}, G_{*_{H}}:[0, T] \times H \times \mathbb{R} \times \mathbb{R}^{m} \times \mathbb{S}^{m} \rightarrow \overline{\mathbb{R}}
$$

be associated with $G$ as follows: for all $(t, x, r, \beta, \gamma) \in(0, T) \times H \times \mathbb{R} \times \mathbb{R}^{m} \times \mathbb{S}^{m}$,

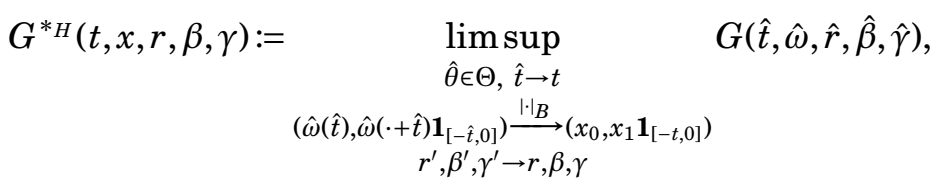

$$
\begin{aligned}
& G_{*_{H}}(t, x, r, \beta, \gamma):=\quad \liminf _{\hat{\theta} \in \Theta, \hat{t} \rightarrow t} \quad G(\hat{t}, \hat{\omega}, \hat{r}, \hat{\beta}, \hat{\gamma}) . \\
& \begin{array}{c}
\left(\hat{\omega}(\hat{t}), \hat{\omega}(\cdot+\hat{t}) \mathbf{1}_{[-\hat{t}, 0]}\right) \\
r^{\prime}, \beta^{\prime}, \gamma^{\prime} \rightarrow r, \beta, \gamma \\
\stackrel{|\cdot|_{B}}{\longrightarrow}\left(x_{0}, x_{1} \mathbf{1}_{[-t, 0]}\right)
\end{array}
\end{aligned}
$$

Similarly, for $u: \Theta \rightarrow \overline{\mathbb{R}}$, define the functions $u^{*_{H}}, u_{*_{H}}:[0, T] \times H \rightarrow \overline{\mathbb{R}}$ by

$$
\begin{aligned}
& u^{* H}(t, x):=\quad \limsup \quad u(\hat{t}, \hat{\omega}), \\
& \begin{array}{l}
\hat{\theta} \in \Theta, \hat{t} \rightarrow t \\
\left.\mathbf{1}_{[-\hat{t}, 0]}\right) \stackrel{\left\|_{B} \mid\right\|_{B}}{\longrightarrow}\left(x_{0}, x_{1} \mathbf{1}_{[-t, 0]}\right)
\end{array} \\
& u_{*_{H}}(t, x):=\quad \quad \liminf _{\hat{\theta} \in \Theta, \hat{t} \rightarrow t} u(\hat{t}, \hat{\omega}) . \\
& \left(\hat{\omega}(\hat{t}), \hat{\omega}(\cdot+\hat{t}) \mathbf{1}_{[-\hat{t}, 0]}\right) \stackrel{|\cdot|_{B}}{\longrightarrow}\left(x_{0}, x_{1} \mathbf{1}_{[-t, 0]}\right)
\end{aligned}
$$

It is clear that the functions $G^{*_{H}}, G_{*_{H}}, u^{*_{H}}, u_{*_{H}}$ are well-defined, because for all $(t, x) \in[0, T] \times H$ we can find a sequence $\left(t, \omega_{n}\right) \in \Theta$ such that $\left(\omega_{n}(t), \omega_{n}(\cdot+t) \mathbf{1}_{[-t, 0]}\right)$ converges to $\left(x_{0}, x_{1} \mathbf{1}_{[-t, 0]}\right)$ in the norm $|\cdot|_{H}$, hence in $|\cdot|_{B}$. 
We will now recall the definition of viscosity solution for PDEs on Hilbert spaces as provided by [12, Ch. 3], and it is in order to fit such a framework that we write (7.8) by emphasizing the maximal dissipative operator $A-I$. Let $\hat{G}:[0, T] \times H \times \mathbb{R} \times \mathbb{R}^{m} \times \mathbb{S}^{m} \rightarrow \overline{\mathbb{R}}$ be a function. Consider the following parabolic equation

$$
-v_{t}-\left\langle(A-I) x, D_{x} v\right\rangle-\left\langle x, D_{x} v\right\rangle-\hat{G}\left(t, x, v, D_{x_{0}} v, D_{x_{0} x_{0}}^{2} v\right)=0 \quad \text { on }(0, T) \times H,
$$

where $v:[0, T] \times H \rightarrow \mathbb{R}$ and where $D_{x_{0}} v, D_{x_{0} x_{0}}^{2} v$ are the first and second order Fréchet differentials of $v$ with respect to the first component $x_{0} \in \mathbb{R}^{m}$ of the variable $x=\left(x_{0}, x_{1}\right) \in H=\mathbb{R}^{m} \times L^{2}\left((0, T), \mathbb{R}^{m}\right)$. It is important to notice that in [12, Ch. 3, Definitions 3.32] some assumptions are stated with respect to topologies induced by an operator denoted by $B$. In our framework, we choose

$$
B:=\left(A^{*}-I\right)^{-1}(A-I)^{-1} \text {. }
$$

Because of the compactness of $(A-I)^{-1}$ (Proposition A.5), hence of $B$, and by [12, Lemma 3.6(i)], in our case the definitions of test function and of viscosity sub-/supersolution given in [12, Ch. 3 , Definitions 3.32 and 3.35] read as follows.

Definition 7.3 (Test functions). A function $\psi:(0, T) \times H \rightarrow \mathbb{R}$ is a test function if $\psi(t, x)=\varphi(t, x)+$ $h\left(t,|x|_{H}\right)$, where:

(i) $\varphi \in C^{1,2}((0, T) \times H, \mathbb{R})$, it is locally bounded, weakly sequentially lower-semicontinuous, $\nabla_{x} \varphi$ takes values in $D\left(A^{*}\right)$, and $\partial_{t} \varphi, A^{*} \nabla_{x} \varphi, \nabla_{x} \varphi, D_{x x}^{2} \varphi$ are uniformly continuous on $(0, T) \times H$;

(ii) $h \in C^{1,2}((0, T) \times \mathbb{R}, \mathbb{R})$ and is such that, for every $t \in(0, T)$, $h(t, \cdot)$ is even and $h(t, \cdot)$ is nondecreasing on $\mathbb{R}^{+}$.

Definition 7.4 ( $H$-viscosity sub-/supersolution). A locally bounded weakly sequentially u.s.c. function $u:(0, T) \times H \rightarrow \mathbb{R}$ is a $H$-viscosity subsolution of (7.8) if, whenever $u-\psi$ has a local maximum at a point $(t, x) \in(0, T) \times H$ for a test function $\psi(s, y)=\varphi(s, y)+h\left(s,|y|_{H}\right)$, then

$$
-\psi_{t}(t, x)-\left\langle x,(A-I)^{*} \nabla_{x} \varphi(t, x)\right\rangle_{H}-\left\langle x, \nabla_{x} \psi(t, x)\right\rangle_{H}-\hat{G}\left(t, x, u(t, x), \nabla_{x_{0}} \psi(t, x), D_{x_{0} x_{0}}^{2} \psi(t, x)\right) \leq 0 .
$$

A locally bounded weakly sequentially l.s.c. function $v:(0, T) \times H \rightarrow \mathbb{R}$ is a viscosity supersolution of (7.8) if, whenever $v+\psi$ has a local minimum at a point $(t, x) \in(0, T) \times H$ for a test function $\psi(s, y)=\varphi(s, y)+h\left(s,|y|_{H}\right)$, then

$$
\psi_{t}(t, x)+\left\langle x,(A-I)^{*} \nabla_{x} \varphi(t, x)\right\rangle+\left\langle x, \nabla_{x} \psi(t, x)\right\rangle-\hat{G}\left(t, x, u(t, x),-\nabla_{x_{0}} \psi(t, x),-D_{x_{0} x_{0}}^{2} \psi(t, x)\right) \geq 0 .
$$

Remark 7.5. The Definition 7.4 does not correspond exactly to [12, Definition 3.35], because we drop the continuity assumption on $\hat{G}$. We will recover such assumption when dealing with comparison.

The first main result of this section is the following

Theorem 7.6. Let $u$ be a $\mathscr{P}_{L}$-viscosity subsolution (resp. supersolution) of (3.1). Then $u^{*_{H}}$ (resp. $u_{*_{H}}$ ) is a H-viscosity subsolution (resp. supersolution) of

$$
\begin{gathered}
-v_{t}-\left\langle(A-I) x, D_{x} v\right\rangle-\left\langle x, D_{x} v\right\rangle-G^{*_{H}}\left(t, x, v, D_{x_{0}} v, D_{x_{0} x_{0}}^{2} v\right)=0 \\
\left(\text { resp. }-v_{t}-\left\langle(A-I) x, D_{x} v\right\rangle-\left\langle x, D_{x} v\right\rangle-G_{*_{H}}\left(t, x, v, D_{x_{0}} v, D_{x_{0} x_{0}}^{2} v\right)=0\right) .
\end{gathered}
$$

Theorem 7.6 allows to exploit the comparison for $H$-viscosity solutions ([12, Theorem 3.50]) to get as corollary a comparison result for $\mathscr{P}_{L}$-viscosity solutions (Corollary 7.7). As a byproduct, we also obtain a sufficient condition for the Perron-type result we proved in Section 5. 
Corollary 7.7. Let $G$ satisfy Assumptions 5.1, with $d=d_{B}$. Let $u$ be a bounded $\mathscr{P}_{L}$-viscosity subsolution of (3.1) and let $v$ be a bounded $\mathscr{P}_{L}$-viscosity supersolution of (3.1). Suppose that

$$
\lim _{r, \eta \rightarrow 0} \sup \left\{u(\theta)-v\left(\theta^{\prime}\right): d_{B}\left(\theta, \theta^{\prime}\right)<r, T-\eta \leq t, t^{\prime} \leq T\right\} \leq 0 .
$$

Then $u \leq v$ on $\Theta$. Moreover, Assumption 5.2 is fulfilled with $\underline{u}:=u, \bar{v}:=v, d:=d_{B}$.

Before proving Theorem 7.6 and Corollary 7.7, we need a preliminary discussion useful to relate the two different settings of $\mathscr{P}_{L}$-viscosity solutions and of viscosity solutions on $H$ as defined by Definition 7.4.

Let $U:[0, T] \times H \rightarrow \mathbb{R}$ be a measurable function such that $U(t, x)=U\left(t, x_{0}, x_{1} \mathbf{1}_{[-t, 0]}\right)$ for $(t, x) \in$ $[0, T] \times H\left(x=\left(x_{0}, x_{1}\right)\right)$. Notice that, for any $(s, x) \in H$, the measurability of $U$ entails the measurability of

$$
\left(\Theta, d_{\infty}\right) \rightarrow \mathbb{R}, \theta \mapsto U^{s, x}(\theta):=U\left((s+t) \wedge T, x_{0}+\omega(s), x_{1} \otimes_{s}\left[x_{0}, \omega\right]\right),
$$

where, for any function $\omega \in \Omega$ and $(s, x) \in[0, T] \times H$,

$$
x_{1} \otimes_{s}\left[x_{0}, \omega\right]:= \begin{cases}x_{1}(t+s) & \forall t \in(-\infty,-s) \\ x_{0}+\omega(t+s) & \forall t \in[-s, 0]\end{cases}
$$

Let $(t, x) \in[0, T] \times H$ and let $U:[0, T] \times H \rightarrow \mathbb{R}$ be a measurable function, locally bounded from above (resp. locally bounded from below). We define the jet $\underline{\mathscr{J}}_{L}^{H} U(t, x)\left(\right.$ resp. $\left.\overline{\mathscr{J}}_{L}^{H} U(t, x)\right)$ by

$$
\begin{gathered}
\mathscr{\mathscr { J }}_{L}^{H} U(t, x):=\left\{(\alpha, \beta, \gamma) \in \mathbb{R} \times \mathbb{R}^{m} \times \mathbb{S}^{m}: U(t, x)=\overline{\mathscr{E}}_{L}\left[\left(U^{t, x}-\varphi^{\alpha, \beta, \gamma}\right)\left(\mathrm{T} \wedge \mathrm{H}_{\delta}, \mathrm{B}\right)\right],\right. \\
\text { for some } \delta \in(0, T-t]\} \\
\left(\text { resp. } \overline{\mathscr{J}}_{L}^{H} U(t, x):=\left\{(\alpha, \beta, \gamma) \in \mathbb{R} \times \mathbb{R}^{m} \times \mathbb{S}^{m}: U(t, x)=\underline{\mathscr{E}}_{L}\left[\left(U^{t, x}-\varphi^{\alpha, \beta, \gamma}\right)\left(\mathrm{T} \wedge \mathrm{H}_{\delta}, \mathrm{B}\right)\right],\right.\right. \\
\text { for some } \delta \in[0, T-t]\}) .
\end{gathered}
$$

Now let $u_{n}$ be a sequence of $d_{\infty}$-u.s.c. functions uniformly bounded from above and let us define a function $\bar{u}^{H}:[0, T] \times H \rightarrow \mathbb{R}\left(\right.$ resp. $\left.\underline{u}_{H}\right)$ by

$$
\begin{aligned}
& \bar{u}^{H}(t, x):=\quad \limsup _{\hat{\theta} \in \Theta} \quad u_{n}(\hat{t}, \hat{\omega}) \quad \forall(t, x) \in[0, T] \times H, \\
& \left(\hat{\omega}(\hat{t}), \hat{\omega}(\cdot+\hat{t}) \mathbf{1}_{[-\hat{t}, 0]} \stackrel{\stackrel{|\cdot| \cdot B}{\longrightarrow} \rightarrow \infty}{\longrightarrow}\left(x_{0}, x_{1} \mathbf{1}_{[-t, 0]}\right)\right.
\end{aligned}
$$

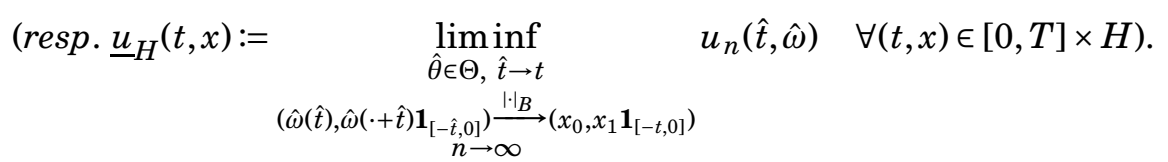

Proposition 7.8. Let $u_{n}, \bar{u}^{H}, \underline{u}^{H}$ be as above. Then for any $(\alpha, \beta, \gamma) \in \underline{\mathscr{J}}_{L}^{H} \bar{u}^{H}(t, x)(\operatorname{resp} .(\alpha, \beta, \gamma) \in$ $\left.\overline{\mathscr{J}}_{L}^{H} \underline{u}_{H}(t, x)\right)$, there exist $\theta_{n} \in \Theta,\left(\alpha_{n}, \beta_{n}, \gamma\right) \in \underline{\mathscr{J}}_{L} u_{n}\left(\theta_{n}\right)$ such that

$$
\begin{gathered}
t_{n} \rightarrow t, \quad\left(\omega_{n}\left(t_{n}\right), \omega_{n}\left(\cdot+t_{n}\right) \mathbf{1}_{\left[-t_{n}, 0\right]}\right) \stackrel{|\cdot|_{B}}{\longrightarrow}\left(x_{0}, x_{1} \mathbf{1}_{[-t, 0]}\right), \\
\text { and }\left(u_{n}\left(\theta_{n}\right), \alpha_{n}, \beta_{n}\right) \rightarrow\left(\bar{u}^{H}(t, x), \alpha, \beta\right) \quad\left(\text { resp. }\left(u_{n}\left(\theta_{n}\right), \alpha_{n}, \beta_{n}\right) \rightarrow\left(\underline{u}_{H}(t, x), \alpha, \beta\right)\right) .
\end{gathered}
$$


Proof. The desired result is very similar to Proposition 4.4 and can be proved with the same argument.

Proposition 7.9. Let u be a $\mathscr{P}_{L}$-viscosity subsolutions to (3.1). Then

$$
-\alpha-G^{*_{H}}\left(t, x, u^{{ }^{H} H}(t, x), \alpha, \beta, \gamma\right) \leq 0, \quad \forall(t, x) \in(0, T) \times H, \forall(\alpha, \beta, \gamma) \in \underline{\mathscr{J}}_{L}^{H} u^{*_{H}}(t, x) .
$$

Proof. Let $(t, x) \in(0, T) \times H$ and $(\alpha, \beta, \gamma) \in \mathscr{J}_{L}^{H} u^{*_{H}}(t, x)$. By the very definition of $u^{*_{H}}$, we can apply Proposition 7.8 to get sequences $\theta_{n} \in \Theta$ and $\left(\alpha_{n}, \beta_{n}\right) \in \mathbb{R} \times \mathbb{R}^{m}$ such that $\left(\alpha_{n}, \beta_{n}, \gamma\right) \in \underline{\mathscr{J}}_{L} u\left(\theta_{n}\right)$ and

$$
t_{n} \rightarrow t, \quad\left(\omega_{n}\left(t_{n}\right), \omega_{n}\left(\cdot+t_{n}\right) \mathbf{1}_{\left[-t_{n}, 0\right]}\right) \stackrel{|\cdot|_{B}}{\longrightarrow}\left(x_{0}, x_{1} \mathbf{1}_{[-t, 0]}\right), \quad\left(u_{n}\left(\theta_{n}\right), \alpha_{n}, \beta_{n}\right) \rightarrow\left(u^{*_{H}}(t, x), \alpha, \beta\right) .
$$

Since $u$ is a $\mathscr{P}_{L}$-viscosity solution to (3.1), we have, for all $n,-\alpha_{n}-G\left(t_{n}, \omega_{n}, u_{n}\left(\theta_{n}\right), \beta_{n}, \gamma\right) \leq 0$. By taking the limit $n \rightarrow \infty$, by using (7.18), and by the very definition of $G^{*}$, we obtain

$$
-\alpha-G^{*_{H}}\left(t, x, u^{*_{H}}(t, x), \alpha, \beta, \gamma\right) \leq 0,
$$

which concludes the proof.

In what follows, for $t \in[0, T]$ and $\mathbb{P} \in \mathscr{P}_{L}$, we denote by $\mathbb{G}^{t}$ the translated filtration $\left\{\mathscr{G}_{0 \vee(s-t)}\right\}_{s \in[0, T]}$ and by $\overline{\mathbb{G}}_{+}^{\mathbb{P}, t}$ its right-continuous $\mathbb{P}$-completion. For $t \in[0, T], x=\left(x_{0}, x_{1}\right) \in H$, we define the $H$ valued process $\mathrm{Z}^{t, x}=\left(\mathrm{Z}_{0}^{t, x}, \mathrm{Z}_{1}^{t, x}\right)$ as follows:

$$
\begin{aligned}
& \text { if } s \in[0, t], \quad \mathrm{Z}_{0, s}^{t, x}:=x_{0} \quad \text { and } \quad \mathrm{Z}_{1, s}^{t, x}:=x_{1} \\
& \text { if } s \in(t, T], \quad \mathrm{Z}_{0, s}^{t, x}:=x_{0}+\mathrm{A}_{s-t}+\mathrm{M}_{s-t} \quad \text { and } \quad \mathrm{Z}_{1, s}^{t, x}(r):= \begin{cases}x_{1}(r+s-t) & r \in(-\infty,-s+t) \\
\mathrm{Z}_{0, r+s}^{t, x} & r \in[-s+t, 0) .\end{cases}
\end{aligned}
$$

We also introduce the following functions

$$
\widetilde{\mathbf{b}}:[0, T] \times \widetilde{\Theta} \rightarrow H \quad \boldsymbol{\sigma}: \widetilde{\Theta} \rightarrow L\left(\mathbb{R}^{m}, H\right)
$$

defined by $\left({ }^{1}\right)$

$$
\widetilde{\mathbf{b}}_{s}(\vartheta):=\left\{\begin{array}{ll}
\left(\dot{a}_{s}, 0\right) & \text { if } a \in W^{1,2}\left([0, T], \mathbb{R}^{m}\right) \\
(0,0) & \text { otherwise }
\end{array} \quad \text { and } \quad \boldsymbol{\sigma}(\vartheta)(v):=(v, 0)\right.
$$

for $\vartheta \in \widetilde{\Theta}, s \in[0, T], v \in \mathbb{R}^{m}$.

Lemma 7.10. Let $Z, \widetilde{\mathbf{b}}$ be as above. Then there exists an $\mathbb{R}^{m}$-valued predictable process $\mathbf{b}$ such that, for all $\mathbb{P} \in \mathscr{P}_{L},\left\{(s, \vartheta) \in[0, T] \times \widetilde{\Theta}: \mathbf{b}_{s}(\vartheta) \neq \widetilde{\mathbf{b}}_{s}(\vartheta)\right\}$ is contained in a Lebesgue $\otimes \mathbb{P}$-null set and

$$
\mathbb{P} \text {-a.s. } \begin{cases}\mathrm{Z}_{s}^{t, x}=x & \forall s \in[0, t] \\ \mathrm{Z}_{s}^{t, x}=S_{s-t} x+\int_{t}^{s} S_{s-r} \mathbf{b}_{r-t} d r+\int_{t}^{s} S_{s-r} \boldsymbol{\sigma} d \mathrm{M}_{r}^{t} & \forall s \in(t, T],\end{cases}
$$

where $\mathrm{M}^{t}:=\left\{\mathrm{M}_{0 \vee(r-t)}\right\}_{r \in[0, T]}$ is a $\left(\overline{\mathbb{G}}_{+}^{\mathbb{P}, t}, \overline{\mathbb{P}}\right)$-martingale.

\footnotetext{
${ }^{1}$ Recall that $\vartheta=((t, \omega), a, \mu, q)$.
} 
Proof. We show the existence of $\mathbf{b}$. First, notice that the function

$$
\rho:[0, T] \times \widetilde{\Theta} \rightarrow L^{2}\left([0, T], \mathbb{R}^{m}\right),(s, \vartheta) \mapsto a_{s \wedge} .
$$

is predictable. By using [13, p. 67 , statement (2)], it is not difficult to show that $W^{1,2}\left([0, T], \mathbb{R}^{m}\right)$ is a Borel subset of $L^{2}\left([0, T], \mathbb{R}^{m}\right)$. Define $B:=\rho^{-1}\left(W^{1,2}\left([0, T], \mathbb{R}^{m}\right)\right)$. It then follows that the map

$$
[0, T] \times \widetilde{\Theta} \rightarrow \mathbb{R},(s, \vartheta) \mapsto \mathbf{1}_{B}(T, \vartheta)
$$

is predictable, and hence also the map

$$
F:[0, T] \times \widetilde{\Theta} \rightarrow \mathbb{R}^{m},(s, \vartheta) \mapsto \mathbf{1}_{B}(T, \vartheta) a_{s}
$$

is predictable. We then obtain the predictability of the left-hand side derivative

$$
B_{1} \rightarrow \mathbb{R}^{m},(s, \vartheta) \mapsto \partial_{s}^{-} F(s, \vartheta),
$$

on the predictable set $B_{1}$ of points $(s, \vartheta)$ where such derivative exists. Since $\mathbb{P} \in \mathscr{P}_{L}, L e b \otimes \mathbb{P}([0, T] \times$ $\left.\widetilde{\Theta} \backslash B_{1}\right)=0$, hence $\mathbf{b}_{s}(\vartheta):=\left(\mathbf{1}_{B_{1}}(s, \vartheta) \partial_{s}^{-} F(s, \vartheta), 0\right) \in H$ satisfies the needed requirements.

Clearly (7.20) holds true if $s \in[0, t]$. We then assume $s \in(t, T]$. Let $\mathbb{P} \in \mathscr{P}_{L}$. For $y \in \mathbb{R}^{m}$, by Itô's formula ([21, Theorem D.2]) and noticing that

$$
\left\langle\left(y_{0}, 0\right), z\right\rangle_{H}=\left\langle\left(y_{0}, 0\right), S_{v} z\right\rangle_{H} \quad \forall z \in H, v \geq 0,
$$

we have, $\mathbb{P}$-a.s.,

$$
\begin{aligned}
\left\langle\left(y_{0}, 0\right), \mathrm{Z}_{s}^{t, x}\right\rangle_{H} & =\left\langle y_{0}, \mathrm{Z}_{0, s}^{t, x}\right\rangle=\left\langle y_{0}, x_{0}\right\rangle+\int_{t}^{s}\left\langle y_{0}, \dot{a}_{r-t}\right\rangle d r+\int_{t}^{s}\left\langle y_{0}, d \mathrm{M}_{r}^{t}\right\rangle \\
& =\left\langle\left(y_{0}, 0\right), x\right\rangle_{H}+\int_{t}^{s}\left\langle\left(y_{0}, 0\right), S_{s-r} \mathbf{b}_{r-t}\right\rangle_{H} d r+\int_{t}^{s}\left\langle\left(y_{0}, 0\right), S_{s-r} \sigma d \mathrm{M}_{r}^{t}\right\rangle_{H} \\
& =\left\langle\left(y_{0}, 0\right), x+\int_{t}^{s} S_{s-r} \mathbf{b}_{r-t} d r+\int_{t}^{s} S_{s-r} \sigma d \mathrm{M}_{r}^{t}\right\rangle_{H} .
\end{aligned}
$$

Now let $y_{1} \in L^{2}\left((-\infty, 0), \mathbb{R}^{m}\right)$. By the very definition of $Z_{s}^{t, x}$, we have, $\mathbb{P}$-a.s.,

$$
\begin{aligned}
\left\langle\left(0, y_{1}\right), \mathrm{Z}_{s}^{t, x}\right\rangle_{H}= & \left\langle y_{1}, \mathrm{Z}_{1, s}^{t, x}\right\rangle_{L^{2}}=\int_{-\infty}^{0}\left\langle y_{1}(v), \mathrm{Z}_{1, s}^{t, x}(v)\right\rangle d v \\
= & \int_{-\infty}^{0}\left\langle y_{1}(v),\left(\mathbf{1}_{(-\infty,-s+t)}(v) x_{1}(v)+\mathbf{1}_{[-s+t, 0)}(v) \mathrm{Z}_{0, v+s}^{t, x}\right)\right\rangle d v \\
= & \int_{-\infty}^{0}\left\langle y_{1}(v),\left(\mathbf{1}_{(-\infty,-s+t)}(v) x_{1}(v)+\mathbf{1}_{[-s+t, 0)}(v) x_{0}\right)\right\rangle d v \\
& +\int_{-\infty}^{0}\left\langle y_{1}(v), \mathbf{1}_{[-s+t, 0)}(v)\left(\int_{t}^{v+s} \dot{a}_{r-t} d r+\int_{t}^{v+s} d \mathrm{M}_{r}^{t}\right)\right\rangle d v=: \mathbf{I}+\mathbf{I I}
\end{aligned}
$$

Now observe that

$$
\mathbf{I}=\left\langle\left(0, y_{1}\right), S_{s-t} x\right\rangle_{H} .
$$

Moreover, by using the stochastic Fubini theorem ([21, Theorem 8.14]) and denoting by $\mathrm{M}_{i}^{t}$ and $y_{1}^{i}$ the $i$ th component of $\mathrm{M}^{t}$ and $y_{1}$, respectively, and by $e_{i}$ the $i$ th element of the canonical basis of $\mathbb{R}^{m}$, we have, $\mathbb{P}$-a.s.,

$$
\begin{aligned}
\mathbf{I I} & =\int_{t}^{s}\left(\int_{-\infty}^{0}\left\langle y_{1}(v), \mathbf{1}_{[r-s, 0)}(v) \dot{a}_{r-t}\right\rangle d v\right) d r+\sum_{i=1, \ldots, m} \int_{t}^{s}\left(\int_{-\infty}^{0} y_{1}^{i}(v) \mathbf{1}_{[r-s, 0)}(v) d v\right) d \mathrm{M}_{i, r}^{t} \\
& =\int_{t}^{s}\left\langle\left(0, y_{1}\right), S_{s-r} \mathbf{b}_{r-t}\right\rangle_{H} d r+\sum_{i=1, \ldots, m} \int_{t}^{s}\left\langle\left(0, y_{1}\right), S_{s-r} \sigma\left(e_{i}\right)\right\rangle_{H} d \mathrm{M}_{i, r}^{t} \\
& =\left\langle\left(0, y_{1}\right), \int_{t}^{s} S_{s-r} \mathbf{b}_{r-t} d r\right\rangle_{H}+\left\langle\left(0, y_{1}\right), \int_{t}^{s} S_{s-r} \sigma d \mathrm{M}_{r}^{t}\right\rangle_{H}
\end{aligned}
$$


By collecting (7.21), (7.22), (7.23), (7.24) and recalling that $y_{0}, y_{1}$ were arbitrarily chosen, we obtain (7.20).

Notice that the $H$-valued process $Z^{t, x}$ defined by (7.19) is independent on $\mathbb{P} \in \mathscr{P}_{L}$ and everywhere continuous, whereas equation (7.20) depends on the chosen $\mathbb{P}$ through the chosen version of the stochastic integral.

Proof of Theorem 7.6. We write the proof only for the subsolution case, since the other case is symmetric.

By the very definition, $u^{*_{H}}$ is $|\cdot|+|\cdot|_{B}$-u.s.c., hence by compactness of $(A-I)^{-1}$ (Proposition A.5) it is weakly sequentially u.s.c. Now let $\psi(s, y)=\varphi(s, y)+h\left(s,|y|_{H}\right)$ be a test function and let $(t, x) \in(0, T) \times H$ be a maximum for $u^{* H}-\psi$ on $[t-\xi, t+\xi] \times B(x ; \xi)$, where $B(x ; \xi)$ denotes the $\left(H,|\cdot|_{H}\right)$-ball centered in $x$ with radius $\xi \in(0, t \wedge(T-t))$.

Notice that $A-1 / 2$ is maximal dissipative. Let $A_{n}:=n A(n-A)^{-1}, n \geq 1$, be the Yosida approximation of $A$. Then there exists $\bar{n} \geq 1$ such that $\left|S_{t}^{n}\right|_{L(H)} \leq e^{t}, t \geq 0, n \geq \bar{n}$, where $S^{n}$ denotes the continuous semigroup generated by $A_{n}$.

For some $\varepsilon \in(0, \xi \wedge(T-t))$ to be chosen later, define the function $\tau_{\varepsilon}: C([0, T], H) \rightarrow[t, T]$ by

$$
\tau_{\varepsilon}(f):=\inf \left\{r \in[t, T]: r-t+\sup _{v \in[t, r]}|f(v)-x|_{H} \geq \varepsilon\right\} \quad \forall f \in C([0, T], H) .
$$

Then $\tau_{\varepsilon}$ is continous. Moreover, for any adapted $H$-valued continuous process $\mathrm{P}, \tau_{\varepsilon}(\mathrm{P})$ is a stopping time.

Now we fix $\mathbb{P} \in \mathscr{P}_{L}$. For $n \geq 1$, define the process $Z_{n}^{t, x}$ by

$$
\begin{cases}\mathrm{Z}_{n, s}^{t, x}:=x & \forall s \in[0, t] \\ \mathrm{Z}_{n, s}^{t, x}:=S_{s-t}^{n} x+\int_{t}^{s} S_{s-r}^{n} \mathbf{b}_{r-t} d r+\int_{t}^{s} S_{s-r}^{n} \boldsymbol{\sigma} d \mathrm{M}_{r}^{t} & \forall s \in(t, T] .\end{cases}
$$

We can choose for $Z_{n}^{t, x}$ a continuous $\left(\overline{\mathbb{G}}_{+}^{\mathbb{P}, t}, \overline{\mathbb{P}}\right)$-version, and we do it (we refer to the discussion in [21, Section 11.4], after recalling that in our case the quadratic variation of $\mathrm{M}$ is bounded). For any $\mathbb{G}^{t}$-stopping time $\rho \geq t$ and for any $f \in C([0, T], H)$, denote

$$
\tau(f):=\tau_{\rho, \varepsilon}(f):=\rho \wedge \tau_{\varepsilon}(f) .
$$

Let $Z$ be defined as in (7.19). Notice that $\tau\left(Z^{t, x}\right)$ is a $\mathbb{G}^{t}$-stopping time and $\tau\left(Z_{n}^{t, x}\right)$ is a $\mathbb{\mathbb { G }}^{\mathbb{P}, t}$-stopping time. For $n \geq 1$, define on $\left(\widetilde{\Theta}, \widetilde{\mathbb{G}}^{\mathbb{P}, t}, \overline{\mathbb{P}}\right)$ the continuous processes $\mathrm{Y}^{t, x}$ and $\mathrm{Y}_{n}^{t, x}$ by

$$
\begin{cases}\mathrm{Y}_{s}^{t, x}:=x & \forall s \in[0, t] \\ \mathrm{Y}_{s}^{t, x}:=S_{s-t} x+\int_{t}^{s} \mathbf{1}_{r<\tau\left(\mathrm{Z}^{t, x}\right)} S_{s-r} \mathbf{b}_{r-t} d r+\int_{t}^{s} \mathbf{1}_{r \leq \tau\left(\mathrm{Z}^{t, x}\right)} S_{s-r} \boldsymbol{\sigma} d \mathrm{M}_{r}^{t} & \forall s \in(t, T],\end{cases}
$$

and

$$
\begin{cases}\mathrm{Y}_{n, s}^{t, x}:=x & \forall s \in[0, t] \\ \mathrm{Y}_{n, s}^{t, x}:=S_{s-t}^{n} x+\int_{t}^{s} \mathbf{1}_{r \leq \tau\left(\mathrm{Z}_{n}^{t, x}\right)} S_{s-r}^{n} \mathbf{b}_{r} d r+\int_{t}^{s} \mathbf{1}_{r \leq \tau\left(\mathrm{Z}_{n}^{t, x}\right)} S_{s-r}^{n} \boldsymbol{\sigma} d \mathrm{M}_{r}^{t} & \forall s \in(t, T] .\end{cases}
$$

Notice that, by Lemma 7.10 and by the very definition of $Z_{n}^{t, x}$, we have, for all $s \in(t, T], n \geq 1$,

$$
\begin{cases}\mathrm{Y}_{s}^{t, x}=\mathrm{Z}_{s}^{t, x} & \overline{\mathbb{P}} \text {-a.s. on }\left\{\tau\left(\mathrm{Z}^{t, x}\right)>s\right\} \\ \mathrm{Y}_{s}^{t, x}=S_{s-\tau\left(\mathrm{Z}^{t, x}\right)} \mathrm{Z}_{\tau\left(\mathrm{Z}^{t, x}\right)}^{t, x} & \mathbb{\mathbb { P }} \text {-a.s. on }\left\{\tau\left(\mathrm{Z}^{t, x}\right) \leq s\right\}\end{cases}
$$




$$
\begin{cases}\mathrm{Y}_{n, s}^{t, x}=\mathrm{Z}_{n, s}^{t, x} & \overline{\mathbb{P}} \text {-a.s. on }\left\{\tau\left(\mathrm{Z}_{n}^{t, x}\right)>s\right\} \\ \mathrm{Y}_{n, s}^{t, x}=S_{s-\tau\left(\mathrm{Z}_{n}^{t, x}\right)}^{n} \mathrm{Z}_{n, \tau\left(\mathrm{Z}_{n}^{t, x}\right)}^{t, x} & \overline{\mathbb{P}} \text {-a.s. on }\left\{\tau\left(\mathrm{Z}_{n}^{t, x}\right) \leq s\right\} .\end{cases}
$$

Now, since $A_{n}$ generates a continuous group in $L(H)$, the process $Y_{n}^{t, x}$ is the strong solution to the following linear SDE in integral form on $\left(\widetilde{\Theta}, \widetilde{\mathbb{G}}^{\mathbb{P}}, t, \widetilde{\mathbb{P}}\right)$ :

$$
\begin{aligned}
\mathrm{Y}_{n, s}^{t, x} & =x+\int_{t}^{s}\left(A_{n} \mathrm{Y}_{n, r}^{t, x}+\mathbf{1}_{r \leq \tau\left(\mathrm{Z}_{n}^{t, x}\right)} \mathbf{b}_{r-t}\right) d r+\int_{t}^{s} \mathbf{1}_{r \leq \tau\left(\mathrm{Z}_{n}^{t, x}\right)} \boldsymbol{\sigma} d \mathrm{M}_{r}^{t} \\
& =x+A_{n} \int_{t}^{s} \mathrm{Y}_{n, r}^{t, x} d r+\boldsymbol{\sigma}\left(\mathrm{A}_{\tau\left(\mathrm{Z}_{n}^{t, x}\right) \wedge s-t}+\mathrm{M}_{\tau\left(\mathrm{Z}_{n}^{t, x}\right) \wedge s-t}\right) \quad \mathbb{P} \text {-a.s., } \forall s \in[t, T) .
\end{aligned}
$$

By (7.30) and (7.31), we have

$$
\begin{array}{rl}
\mathrm{Z}_{n, \tau\left(\mathrm{Z}_{n}^{t, x}\right)}^{t, x} & x+\int_{t}^{\tau\left(\mathrm{Z}_{n}^{t, x}\right)}\left(A_{n} \mathrm{Z}_{n, r}^{t, x}+\mathbf{b}_{r-t}\right) d r+\int_{t}^{\tau\left(\mathrm{Z}_{n}^{t, x}\right)} \boldsymbol{\sigma} d \mathrm{M}_{r}^{t} \\
& =x+A_{n} \int_{t}^{\tau\left(\mathrm{Z}_{n}^{t, x}\right)} \mathrm{Z}_{n, r}^{t, x} d r+\boldsymbol{\sigma}\left(\mathrm{A}_{\tau\left(\mathrm{Z}_{n}^{t, x}\right)-t}+\mathrm{M}_{\tau\left(\mathrm{Z}_{n}^{t, x}\right)-t}\right) \quad \text { P-a.s., } \forall s \in[t, T) .
\end{array}
$$

By the assumptions on the test function $\psi=\varphi+h$, by using (7.32), and by recalling that $\left|\mathrm{Z}_{n, \tau\left(\mathrm{Z}_{n}^{t, x}\right) \wedge \cdot}^{t, x}-x\right|_{\infty} \leq \varepsilon$ for all $n$, we can apply Itô's formula ([21, Theorem D.2]) to $\psi\left(\tau\left(\mathrm{Z}_{n}^{t, x}\right), \mathrm{Z}_{n, \tau}^{t, x}\left(\mathrm{Z}_{n}^{t, x}\right)\right.$ and take the expectation, to get, for $n \geq \bar{n}$,

$$
\begin{aligned}
\mathbb{E}^{\bar{P}}\left[\psi\left(\tau\left(\mathrm{Z}_{n}^{t, x}\right), \mathrm{Z}_{n, \tau\left(\mathrm{Z}_{n}^{t, x}\right)}^{t, x}\right)=\right. & \psi(t, x)+\mathbb{E}^{\bar{P}}\left[\int_{t}^{\tau\left(\mathrm{Z}_{n}^{t, x}\right)}\left(\partial_{t} \psi\left(r, \mathrm{Z}_{n, r}^{t, x}\right)+\left\langle\nabla_{x} \psi\left(r, \mathrm{Z}_{n, r}^{t, x}\right), A_{n} \mathrm{Z}_{n, s}^{t, x}\right\rangle\right) d r\right] \\
& +\mathbb{E}^{\overline{\mathbb{P}}}\left[\int_{t}^{\tau\left(\mathrm{Z}_{n}^{t, x}\right)}\left\langle\nabla_{x} \psi\left(r, \mathrm{Z}_{n, r}^{t, x}\right), \mathbf{b}_{r-t}\right\rangle d r\right] \\
& +\frac{1}{2} \mathbb{E}^{\bar{P}}\left[\int_{t}^{\tau\left(\mathrm{Z}_{n}^{t, x}\right)} \operatorname{Tr}\left[\boldsymbol{\sigma} \dot{\mathrm{Q}}_{r-t} \boldsymbol{\sigma}^{*} D_{x x}^{2} \psi\left(r, \mathrm{Z}_{n, r}^{t, x}\right)\right] d r\right] \\
\leq & \psi(t, x)+\mathbb{E}^{\bar{P}}\left[\int_{t}^{\tau\left(\mathrm{Z}_{n}^{t, x}\right)}\left(\partial_{t} \psi\left(r, \mathrm{Z}_{n, r}^{t, x}\right)+\left\langle A_{n}^{*} \nabla_{x} \varphi\left(r, \mathrm{Z}_{n, r}^{t, x}\right), \mathrm{Z}_{n, r}^{t, x}\right\rangle\right) d r\right] \\
& +\mathbb{E}^{\bar{P}}\left[\int_{t}^{\tau\left(\mathrm{Z}_{n}^{t, x}\right)}\left\langle\nabla_{x} h\left(r, \mathrm{Z}_{n, r}^{t, x}\right), \mathrm{Z}_{n, r}^{t, x}\right\rangle d r\right] \\
& +\mathbb{E}^{\overline{\mathbb{P}}}\left[\int_{t}^{\tau\left(\mathrm{Z}_{n}^{t, x}\right)}\left\langle\nabla_{x} \psi\left(r, \mathrm{Z}_{n, r}^{t, x}\right), \mathbf{b}_{r-t}\right\rangle d r\right] \\
& +\frac{1}{2} \mathbb{E}^{\bar{P}}\left[\int_{t}^{\tau\left(\mathrm{Z}_{n}^{t, x}\right)} \operatorname{Tr}\left[\dot{\mathrm{Q}}_{r-t} D_{x_{0} x_{0}}^{2} \psi\left(r, \mathrm{Z}_{n, r}^{t, x}\right)\right] d r\right],
\end{aligned}
$$

where we have used the fact that $h$ is radial and that

$$
\left\langle A_{n} y, y\right\rangle \leq|y|_{H}^{2} \quad \forall y \in H, n \geq \bar{n} .
$$

We will now take in consideration each term appearing in the formula above and pass to the limit as $n \rightarrow \infty$ to get a useful inequality for $\mathbb{E}\left[\psi\left(\tau\left(Z_{n}^{t, x}\right), Z_{\tau\left(Z_{n}^{t, x}\right)}^{t, x}\right)\right]$. Using (7.20), (7.25), and the standard machinery based on the factorization formula for stochastic convolutions with $C_{0}$-semigroups (see [21, Sections 11.3-4] and recall that the quadratic variation of $M$ is bounded in our case) one can see that

$$
\lim _{n \rightarrow \infty} \mathbb{E}^{\mathbb{P}}\left[\left|Z^{t, x}-Z_{n}^{t, x}\right|_{\infty}^{2}\right]=0
$$


This entails immediately the two following facts

$$
\begin{aligned}
& \lim _{n \rightarrow \infty} \mathbb{\mathbb { E }}^{\overline{\mathbb{P}}}\left[\left|Z_{\tau\left(Z_{n}^{t, x}\right)}^{t, x}-Z_{n, \tau\left(Z_{n}^{t, x}\right)}^{t, x}\right|_{\infty}^{2}\right]=0, \\
& \lim _{n \rightarrow \infty} \tau_{\varepsilon}\left(Z_{n}^{t, x}\right)=\tau_{\varepsilon}\left(Z^{t, x}\right) \quad \overline{\mathbb{P}}_{\text {-a.s. }}
\end{aligned}
$$

By (7.35a) and (7.35b) it follows that

$$
\lim _{n \rightarrow \infty} \mathbb{E}^{\bar{P}}\left[\left|\mathrm{Z}_{\tau\left(\mathrm{Z}^{t, x}\right)}^{t, x}-\mathrm{Z}_{n, \tau\left(\mathrm{Z}_{n}^{t, x}\right)}^{t, x}\right|_{\infty}^{p}\right]=0,
$$

for any $p \in[1,2)$. By the assumptions on $\psi$ and by (7.36) we can then pass to the limit in (7.33) and obtain,

$$
\begin{aligned}
\mathbb{E}^{\mathbb{P}}\left[\psi\left(\tau\left(\mathrm{Z}^{t, x}\right), \mathrm{Z}_{\tau\left(\mathrm{Z}^{t, x}\right)}^{t, x}\right)\right]= & \lim _{n \rightarrow \infty} \mathbb{E}^{\bar{P}}\left[\psi\left(\tau\left(\mathrm{Z}_{n}^{t, x}\right), \mathrm{Z}_{n, \tau\left(\mathrm{Z}^{t, x}\right)}^{t, x}\right)\right] \\
\leq & \psi(t, x)+\mathbb{E}^{\bar{P}}\left[\left(\int_{t}^{\tau\left(\mathrm{Z}^{t, x}\right)} \partial_{t} \psi\left(r, \mathrm{Z}_{r}^{t, x}\right)+\left\langle A^{*} \nabla_{x} \varphi\left(r, \mathrm{Z}_{r}^{t, x}\right), \mathrm{Z}_{r}^{t, x}\right\rangle\right) d r\right] \\
& +\mathbb{E}^{\overline{\mathbb{P}}}\left[\int_{t}^{\tau\left(\mathrm{Z}^{t, x}\right)}\left(\left\langle\nabla_{x} h\left(r, \mathrm{Z}_{r}^{t, x}\right), \mathrm{Z}_{r}^{t, x}\right\rangle+\left\langle\nabla_{x} \psi\left(r, \mathrm{Z}_{r}^{t, x}\right), \mathbf{b}_{r-t}\right\rangle\right) d r\right] \\
& +\frac{1}{2} \mathbb{E}^{\bar{P}}\left[\int_{t}^{\tau\left(\mathrm{Z}^{t, x}\right)} \operatorname{Tr}\left[\dot{\mathrm{Q}}_{r-t} D_{x_{0} x_{0}}^{2} \psi\left(r, \mathrm{Z}_{r}^{t, x}\right)\right] d r\right] .
\end{aligned}
$$

Since $\overline{\mathbb{P}}$ was arbitrary, (7.37) holds for all $\overline{\mathbb{P}} \in \mathscr{P}_{L}$. Now, since $\partial_{t} \psi, A^{*} \nabla_{x} \varphi, \nabla_{x} h, \nabla_{x} \psi, D_{x_{0} x_{0}}^{2} \psi$ are uniformly continuous on bounded sets, recalling the definition of $\tau=\tau_{\rho, \varepsilon}$ in (7.26), and noticing that $\varepsilon \downarrow 0$ implies $\tau_{\varepsilon}\left(\mathrm{Z}^{t, x}\right) \downarrow t \overline{\mathbb{P}}$-a.s., one can easily see that

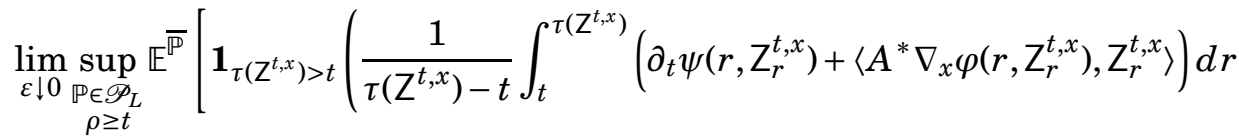

$$
\begin{aligned}
& \left.\left.-\partial_{t} \psi(t, x)+\left\langle A^{*} \nabla_{x} \varphi(t, x), x\right\rangle\right)\right]=0
\end{aligned}
$$

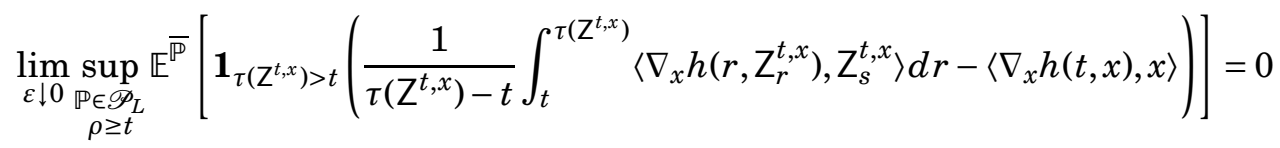

$$
\begin{aligned}
& \lim _{\varepsilon \downarrow 0} \sup _{\substack{\mathbb{P} \in \mathscr{P} \\
\rho \geq t}} \mathbb{E}^{\mathbb{P}}\left[\frac{\mathbf{1}_{\tau\left(Z^{t, x}\right)>t}}{\tau\left(Z^{t, x}\right)-t}\left(\int_{t}^{\tau\left(\mathrm{Z}^{t, x}\right)}\left\langle\nabla_{x} \psi\left(r, Z_{r}^{t, x}\right), \mathbf{b}_{r-t}\right\rangle d r-\left\langle\nabla_{x} \psi(t, x), \mathrm{A}_{\tau\left(\mathrm{Z}^{t, x}\right)-t}\right\rangle\right)\right]=0 \\
& \lim _{\varepsilon \downarrow 0} \sup _{\substack{\mathbb{P} \in \mathscr{P}_{L} \\
\rho \geq t}} \mathbb{E}^{\bar{P}}\left[\frac{\mathbf{1}_{\tau\left(\mathrm{Z}^{t, x}\right)>t}}{\tau\left(\mathrm{Z}^{t, x}\right)-t}\left(\int_{t}^{\tau\left(\mathrm{Z}^{t, x}\right)} \operatorname{Tr}\left[\dot{\mathrm{Q}}_{r} D_{x_{0} x_{0}}^{2} \psi\left(r, Z_{r}^{t, x}\right)\right] d r-\operatorname{Tr}\left[\mathrm{Q}_{\tau\left(\mathrm{Z}^{t, x}\right)-t} D_{x_{0} x_{0}}^{2} \psi(t, x)\right]\right)\right]=0 .
\end{aligned}
$$

Then, for any arbitrarily small real number $\zeta>0$, there exists $\varepsilon$ such that, for all $\mathbb{P} \in \mathscr{P}_{L}$ and all $\mathbb{G}^{t}$-stopping time $\rho \geq t$,

$$
\begin{aligned}
\mathbb{E}^{\mathbb{P}}\left[\int_{t}^{\tau\left(\mathrm{Z}^{t, x}\right)}\left(\partial_{t} \psi\left(r, \mathrm{Z}_{r}^{t, x}\right)+\left\langle A^{*} \nabla_{x} \varphi\left(r, \mathrm{Z}_{r}^{t, x}\right), \mathrm{Z}_{r}^{t, x}\right\rangle\right) d r\right] \\
\leq \mathbb{E}^{\mathbb{P}}\left[\left(\partial_{t} \psi(t, x)+\left\langle A^{*} \nabla_{x} \varphi(t, x), x\right\rangle+\zeta\right)\left(\tau\left(\mathrm{Z}^{t, x}\right)-t\right)\right]
\end{aligned}
$$




$$
\begin{array}{r}
\mathbb{E}^{\mathbb{P}}\left[\int_{t}^{\tau\left(\mathrm{Z}^{t, x}\right)}\left\langle\nabla_{x} h\left(r, \mathrm{Z}_{r}^{t, x}\right), \mathrm{Z}_{s}^{t, x}\right\rangle d r\right] \leq \mathbb{E}^{\mathbb{P}}\left[\left(\left\langle\nabla_{x} h(t, x), x\right\rangle+\zeta\right)\left(\tau\left(\mathrm{Z}^{t, x}\right)-t\right)\right] \\
\mathbb{E}^{\mathbb{P}}\left[\int_{t}^{\tau\left(\mathrm{Z}^{t, x}\right)}\left\langle\nabla_{x} \psi\left(r, \mathrm{Z}_{r}^{t, x}\right), \mathbf{b}_{r-t}\right\rangle d r\right] \leq \mathbb{E}^{\mathbb{P}}\left[\left\langle\nabla_{x} \psi(t, x), \mathrm{A}_{\tau\left(\mathrm{Z}^{t, x}\right)-t}\right\rangle+\zeta\left(\tau\left(\mathrm{Z}^{t, x}\right)-t\right)\right] \\
=\mathbb{E}^{\mathbb{P}}\left[\left\langle\nabla_{x} \psi(t, x), \mathrm{B}_{\tau\left(\mathrm{Z}^{t, x}\right)-t}\right\rangle+\zeta\left(\tau\left(\mathrm{Z}^{t, x}\right)-t\right)\right] \\
\mathbb{E}^{\bar{P}}\left[\int_{t}^{\tau\left(\mathrm{Z}^{t, x}\right)} \operatorname{Tr}\left[\dot{Q}_{r} D_{x_{0} x_{0}}^{2} \psi\left(r, \mathrm{Z}_{r}^{t, x}\right)\right] d r\right] \leq \mathbb{E}^{\bar{P}}\left[\operatorname{Tr}\left[\mathrm{Q}_{\tau\left(\mathrm{Z}^{t, x}\right)-t} D_{x_{0} x_{0}}^{2} \psi(t, x)\right]+\zeta\left(\tau\left(\mathrm{Z}^{t, x}\right)-t\right)\right] .
\end{array}
$$

By defining

$$
\alpha:=\partial_{t} \psi(t, x)+\left\langle\left(A^{*}-I\right) \nabla_{x} \varphi(t, x), x\right\rangle+\left\langle\nabla_{x} \psi(t, x), x\right\rangle+4 \zeta, \quad \beta:=\nabla_{x} \psi(t, x), \quad \gamma:=D_{x_{0} x_{0}}^{2} \psi(t, x),
$$

by recalling that $(t, x)$ is a maximum for $u^{*_{H}}-\psi$ on $[t-\xi, t+\xi] \times B(x ; \xi)$, and by collecting (7.37), (7.38a), (7.38b), (7.38c), (7.38d), we obtain, for all $\mathbb{P} \in \mathscr{P}_{L}$ and all $\rho \geq t$,

$$
u^{* H}(t, x) \geq \mathbb{E}^{\mathbb{P}}\left[u^{* H}\left(\tau\left(\mathrm{Z}^{t, x}\right), \mathrm{Z}_{\tau\left(\mathrm{Z}^{t, x}\right)}^{t, x}\right)-\alpha\left(\tau\left(\mathrm{Z}^{t, x}\right)-t\right)-\left\langle\beta, \mathrm{B}_{\tau\left(\mathrm{Z}^{t, x}\right)-t}\right\rangle-\frac{1}{2}\left\langle\gamma \mathrm{M}_{\tau\left(\mathrm{Z}^{t, x}\right)-t}, \mathrm{M}_{\tau\left(\mathrm{Z}^{t, x}\right)-t}\right\rangle\right] .
$$

We can finally conclude the proof. By recalling the definition of the stopping time $\mathrm{H}_{\delta}$ in (3.3) and by the very definition of $Z^{t, x}$ in (7.19), we see that we can choose $\delta^{*}$ such that

$$
t+\mathrm{H}_{\delta^{*}}(\vartheta) \leq \tau_{\varepsilon}\left(\mathrm{Z}^{t, x}(\vartheta)\right) \quad \forall \vartheta \in \widetilde{\Theta} .
$$

If we now define $\rho:=t+\mathrm{T} \wedge \mathrm{H}_{\delta^{*}}$, we get $\tau\left(\mathrm{Z}^{t, x}\right)=\rho \wedge \tau_{\varepsilon}\left(\mathrm{Z}^{t, x}\right)=t+\mathrm{T} \wedge \mathrm{H}_{\delta^{*}}$ and then (7.39) provides

$$
u^{*_{H}}(t, x)=\overline{\mathscr{E}}_{L}\left[\left(u^{* H}\right)^{t, x}\left(\mathrm{~T} \wedge \mathrm{H}_{\delta^{*}}, \mathrm{~B}\right)-\alpha \mathrm{T} \wedge \mathrm{H}_{\delta^{*}}-\left\langle\beta, \mathrm{B}_{\mathrm{T} \wedge \mathrm{H}_{\delta^{*}}}\right\rangle-\frac{1}{2}\left\langle\gamma \mathrm{M}_{\mathrm{T} \wedge \mathrm{H}_{\delta^{*}}}, \mathrm{M}_{\mathrm{T} \wedge \mathrm{H}_{\delta^{*}}}\right\rangle\right],
$$

where we have used the equality

$$
u^{* H}\left(t+\mathrm{T} \wedge \mathrm{H}_{\delta^{*}} \mathrm{Z}_{t+\mathrm{T} \wedge \mathrm{H}_{\delta^{*}}}\right)=\left(u^{* H}\right)^{t, x}\left(\mathrm{~T} \wedge \mathrm{H}_{\delta^{*}}, \mathrm{~B}\right)
$$

(recall (7.13) for the definition of $\left.U^{s, y_{0}, y_{1}}\right)$. By (7.40) we have that $(\alpha, \beta, \gamma) \in \mathscr{J}_{L}^{H} u^{* H}(t, x)$, hence, by Proposition 7.9 , we obtain $-\alpha-G^{{ }^{*} H}\left(t, x, u^{*_{H}}(t, x), \alpha, \beta, \gamma\right) \leq 0$, or, equivalently,

$$
-\partial_{t} \psi(t, x)-\left\langle\left(A^{*}-I\right) \nabla_{x} \varphi(t, x), x\right\rangle-\left\langle\nabla_{x} \psi(t, x), x\right\rangle-4 \zeta-G^{*_{H}}\left(t, x, u^{*_{H}}(t, x), \nabla_{x} \psi(t, x), D_{x_{0} x_{0}}^{2} \psi(t, x)\right) \leq 0 .
$$

We can now conclude by letting $\zeta$ tends to 0 .

Proof of Corollary 7.7. First notice that, by Theorem 7.6, $u^{*_{H}}$ and $v_{*_{H}}$ are sub- and supersolutions of

$$
-u_{t}-\left\langle(A-I) x, D_{x} u\right\rangle-\left\langle x, D_{x} u\right\rangle-\hat{G}\left(t, x, u, D_{x_{0}} u, D_{x_{0} x_{0}}^{2} u\right)=0
$$

where $\hat{G}:=G^{*_{H}}=G_{*_{H}}$ is well-defined due to Assumption 5.1(i). We want to show that [12, Theorem 3.50] applies to $u^{*_{H}}, v_{*_{H}}$, from which the corollary follows immediately. But it is easy to see that Assumptions 5.1 imply that Hypotheses 3.44-3.49 in [12] are verified with

$$
F(t, x, r, \beta, \gamma):=-\langle x, \beta\rangle-\hat{G}(t, x, r, \beta, \gamma),
$$

where $\hat{G}:=G^{*_{H}}=G_{*_{H}}$. In particular, the nuclearity condition Hypothesis 3.47 is automatically satisfied in our case because the second order term in $\hat{G}$ is finite dimensional, and Hypothesis 3.48 comes from the ellipticity condition (Assumption 5.1(iii)), from the local uniform continuity (Assumption 5.1(i)), and from the Lipschitz continuity of $G$ (Assumption 5.1(iv)). Finally, condition (3.72) in [12, p. 206], with $u, v$ replaced by our $u^{*_{H}}, v_{*_{H}}$, follows by (7.12). 


\section{A Appendix}

We first address some properties of $\mathscr{P}_{L}$. Let $\gamma \in(0,1], \ell>0$. Define

$$
\begin{aligned}
\widetilde{\Theta}_{L}^{\gamma, \ell}:=\{\vartheta \in \widetilde{\Theta}: t \in & {[0, T], \omega=a+\mu, } \\
& \left.\left|a_{s}-a_{s^{\prime}}\right| \leq L\left|s-s^{\prime}\right|,\left|\mu_{s}-\mu_{s^{\prime}}\right| \leq \ell\left|s-s^{\prime}\right|^{\gamma},\left|q_{s}-q_{s^{\prime}}\right| \leq L\left|s-s^{\prime}\right|, \forall s, s^{\prime} \in[0, T]\right\} .
\end{aligned}
$$

Remark A.1. By applying Ascoli-Arzelà theorem, we see that $\mathrm{A}\left(\widetilde{\Theta}_{L}^{\gamma, \ell}\right)$ and $\mathrm{M}\left(\widetilde{\Theta}_{L}^{\gamma, \ell}\right)$ are relatively compact in $\Omega$, and $\mathrm{Q}\left(\widetilde{\Theta}_{L}^{\gamma, \ell}\right)$ is relatively compact in $C_{0}\left([0,1], \mathbb{S}^{m}\right)$. Hence also $\mathrm{B}\left(\widetilde{\Theta}_{L}^{\gamma, \ell}\right)$ is relatively compact. Since $\widetilde{\Theta}_{L}^{\gamma, \ell}$ is closed in $\widetilde{\Theta}$, we conclude that $\widetilde{\Theta}_{L}^{\gamma, \ell}$ is compact in $\widetilde{\Theta}$.

Lemma A.2. For all $\gamma \in\left(0, \frac{1}{2}\right)$, and $\varepsilon>0$, there exists $\ell>0$ such that

$$
\inf _{\mathbb{P} \in \mathscr{P}_{L}} \mathbb{P}\left(\widetilde{\Theta}_{L}^{\gamma, \ell}\right) \geq 1-\varepsilon
$$

Proof. Notice that, if $a \in \mathrm{A}\left(\widetilde{\Theta}_{L}\right)$ and $q \in \mathrm{Q}\left(\widetilde{\Theta}_{L}\right)$, where $\widetilde{\Theta}_{L}$ is defined in (2.1), then $\left|a_{s}-a_{s^{\prime}}\right| \leq L\left|s-s^{\prime}\right|$ and $\left|q_{s}-q_{s^{\prime}}\right| \leq L\left|s-s^{\prime}\right|$, for all $s, s^{\prime} \in[0, T]$. Moreover, since by definition $\mathbb{P}\left(\widetilde{\Theta}_{L}\right)=1$ for all $\mathbb{P} \in \mathscr{P}_{L}$, we only need to show that there exists $\ell>0$ such that

$$
\inf _{\mathbb{P} \in \mathscr{P}_{L}} \mathbb{P}\left(\bar{\Theta}^{\gamma, \ell}\right) \geq 1-\varepsilon
$$

where $\bar{\Theta}^{\gamma, \ell}:=\left\{\vartheta \in \widetilde{\Theta}:\left|\mu_{s}-\mu_{s^{\prime}}\right| \leq \ell\left|s-s^{\prime}\right|^{\gamma}, \forall s, s^{\prime} \in[0, T]\right\}$. But now (A.2) follows by the KolmogorovČntsov continuity theorem ([15, Ch. 2, Theorem 2.8]), after observing by inspection that the proof of the theorem holds uniformly in the reference probability.

Proof of Proposition 4.1. By recalling that $\widetilde{\Theta}_{L}^{\gamma, \ell}$ is compact (Remark A.1), and by applying Prokhorov theorem taking into account Lemma A.2, we obtain that $\mathscr{P}_{L}$ is tight. To conclude the proof, it remains to show that $\mathscr{P}_{L}$ is closed. Let $\left\{\mathbb{P}_{n}\right\}_{n \in \mathbb{N}} \subset \mathscr{P}_{L}$ be a sequence converging to $\mathbb{P}$ in $\mathscr{P}$. We need to show that $\mathbb{P} \in \mathscr{P}_{L}$, i.e.,

$$
\mathbb{P}\left(\widetilde{\Theta}_{L}\right)=1, \quad M \text { is a } \mathbb{P} \text {-martingale, } \quad\langle\mathrm{M}\rangle=\mathrm{Q} \quad \mathbb{P} \text {-a.s. }
$$

Step 1. We show that $\mathbb{P}\left(\widetilde{\Theta}_{L}\right)=1$. We first notice that $\widetilde{\Theta}_{L}$ is closed. So by weak convergence of $\overline{\left\{\mathbb{P}_{n}\right\}_{n \in \mathbb{N}}}$ to $\mathbb{P}$, we have

$$
\mathbb{P}\left(\widetilde{\Theta}_{L}\right) \geq \limsup _{n \rightarrow \infty} \mathbb{P}_{n}\left(\widetilde{\Theta}_{L}\right)=1 .
$$

Step 2. We show that $\mathrm{M}$ is a $\mathbb{P}$-martingale. First, notice that the process $|\mathrm{M}|_{\infty}$ is continuous and that (for some constants $c_{1}>0$ )

$$
\mathbb{E}^{\mathbb{P}_{n}}|\mathrm{M}|_{\infty} \leq c_{1} \mathbb{E}^{\mathbb{P}_{n}}\left[\left(\int_{0}^{T}\left|\dot{Q}_{r}\right| d r\right)^{1 / 2}\right] \leq c_{1} T^{1 / 2} L^{1 / 2} \quad \text { for all } n \in \mathbb{N} .
$$

Hence, by weak convergence, we have

$$
\mathbb{E}^{\mathbb{P}}|\mathrm{M}|_{\infty}=\lim _{j \rightarrow \infty} \mathbb{E}^{\mathbb{P}}\left[j \wedge|\mathrm{M}|_{\infty}\right]=\lim _{j \rightarrow \infty} \lim _{n \rightarrow \infty} \mathbb{E}^{\mathbb{P}_{n}}\left[j \wedge|\mathrm{M}|_{\infty}\right] \leq c_{1} T^{1 / 2} L^{1 / 2}
$$

Let now $\tau_{k}(\vartheta):=\inf \left\{s \in[0, T]: s+\left|\mu_{s \wedge} \cdot\right|_{\infty} \geq k\right\} \wedge t$. Notice that $\tau_{k}$ is continuous on $\widetilde{\Theta}$ and that it is an $\mathbb{G}$-stopping time. Hence $\mathrm{M}_{\tau_{k}}$ is continuous and bounded on $\widetilde{\Theta}$. Since $\mathrm{M}$ is a $\mathbb{P}_{n}$-martingale 
for all $n$, we can write, for all $t, t^{\prime} \in[0, T], t^{\prime}<t, \varphi$ bounded continuous $\mathscr{G}_{t^{\prime}}$-measurable functions, using the weak convergence of $\left\{\mathbb{P}_{n}\right\}_{n}$,

$$
\mathbb{E}^{\mathbb{P}}\left[\varphi \mathrm{M}_{\tau_{k} \wedge t^{\prime}}\right]=\lim _{n \rightarrow \infty} \mathbb{E}^{\mathbb{P}_{n}}\left[\varphi \mathrm{M}_{\tau_{k} \wedge t^{\prime}}\right]=\lim _{n \rightarrow \infty} \mathbb{E}^{\mathbb{P}_{n}}\left[\varphi \mathrm{M}_{\tau_{k} \wedge t}\right]=\mathbb{E}^{\mathbb{P}}\left[\varphi \mathrm{M}_{\tau_{k} \wedge t}\right]
$$

This shows that $\mathrm{M}_{\tau_{k} \wedge}$. is a $\mathbb{P}$-martingale. Due to the fact that $\tau_{k} / t$ pointwise as $k \rightarrow \infty$, we obtain, by passing to the limit in (A.4) after recalling (A.3),

$$
\mathbb{E}^{\mathbb{P}}\left[\varphi \mathrm{M}_{t^{\prime}}\right]=\mathbb{E}^{\mathbb{P}}\left[\varphi \mathrm{M}_{t}\right]
$$

and hence we conclude that $M$ is a $\mathbb{P}$-martingale.

Step 3. We show that $\langle\mathrm{M}\rangle=\mathrm{Q} \mathbb{P}$-a.s. To this aim, it is sufficient to show that $\mathrm{M}^{(i)} \mathrm{M}^{(j)}-\mathrm{Q}^{(i, j)}$ is a PP-martingale, where $\mathrm{M}^{(i)}$ denotes the $i$ th component of the vector $\mathrm{M}$ and $\mathrm{Q}^{(i, j)}$ denotes the $(i, j)$ entry of the matrix Q. After noting that that (for some $c_{2}>0$ )

$$
\mathbb{E}^{\mathbb{P}_{n}}\left[\left|\mathrm{M}^{(i)} \mathrm{M}^{(j)}-\mathrm{Q}^{(i, j)}\right|_{\infty}\right] \leq c_{2} \mathbb{E}^{\mathbb{P}_{n}}\left[\int_{0}^{T}|\dot{\mathrm{Q}}|_{r} d r\right] \leq c_{2} T L \quad \forall n \in \mathbb{N}
$$

we can proceed exactly as in Step 2 .

In the following discussion, we aim to prove the dynamic programming result stated in Proposition 4.3 .

Proof of Lemma 4.2. We need to show that

$$
\mathbb{P}^{*} \in \mathscr{P}_{L} \quad \text { and } \quad \mathrm{T} \leq T-\tau\left(\vartheta^{*}\right), \mathbb{P}^{*} \text {-a.s. }
$$

The former is due to the result of Proposition 4.1, so it remains to prove the latter. Since $\mathbb{P}^{n} \rightarrow \mathbb{P}^{*}$, by Skorohod representation there exists a probability space $(\widehat{\Omega}, \widehat{\mathscr{G}}, \widehat{\mathbb{P}})$ on which

$$
\exists \quad \text { r.v.'s }\left.\left.\quad \mathrm{X}^{n}\right|_{\widehat{\mathbb{P}}} \stackrel{d}{=} \mathrm{X}\right|_{\mathbb{P} n},\left.\left.\quad \mathrm{X}^{*}\right|_{\widehat{\mathbb{P}}} \stackrel{d}{=} \mathrm{X}\right|_{\mathbb{P}^{*}} \text { such that }\left|\mathrm{X}^{n}-\mathrm{X}^{*}\right|_{\infty} \rightarrow 0, \quad \widehat{\mathbb{P}} \text {-a.s., }
$$

where $\stackrel{d}{=}$ denotes equality in distribution. Since $\mathbb{P}^{n} \in \mathscr{P}_{L}\left(\tau, \vartheta^{n}\right)$ and $\tau$ is continuous, it follows from Fatou's lemma that

$$
1=\limsup _{n \rightarrow \infty} \widehat{\mathbb{P}}\left[\mathrm{T}^{n} \leq T-\tau\left(\vartheta^{n}\right)\right] \leq \widehat{\mathbb{P}}\left[\mathrm{T} \leq T-\tau\left(\vartheta^{*}\right)\right]=\mathbb{P}^{*}\left[\mathrm{~T} \leq T-\tau\left(\vartheta^{*}\right)\right],
$$

which concludes the proof.

Proposition A.3. Let $f: \Theta \rightarrow \mathbb{R}$ be $d_{\infty}$-u.s.c. and bounded from above, and $\tau$ be a continuous G-stopping time. Define

$$
V(\vartheta):=\overline{\mathscr{E}}_{L}^{\tau}\left[f\left(\mathrm{~T}^{\tau, \vartheta}, \mathrm{B}^{\tau, \vartheta}\right)\right](\vartheta)=\overline{\mathscr{E}}_{L}^{\tau}\left[f^{t \wedge \tau(\vartheta), \omega}\right](\vartheta) .
$$

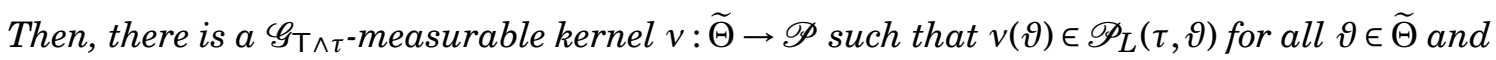

$$
V(\vartheta)=\mathbb{E}^{v(\vartheta)}\left[f^{t \wedge \tau(\vartheta), \omega}\right]
$$


Proof. First note that the equality in (A.6) follows from Remark 2.3. Consider the set $\mathscr{D} \subset \widetilde{\Theta} \times \mathscr{P}_{L}$ defined by $\mathscr{D}:=\left\{(\vartheta, \mathbb{P}): \vartheta \in \widetilde{\Theta}, \mathbb{P} \in \mathscr{P}_{L}(\tau, \vartheta)\right\}$. For all $\mathbb{P} \in \mathscr{P}_{L}(\tau, \vartheta)$ define $\bar{f}: \mathscr{D} \rightarrow \overline{\mathbb{R}}$ by

$$
\bar{f}(\vartheta, \mathbb{P}):=\mathbb{E}^{\mathbb{P}}\left[f^{t \wedge \tau(\vartheta), \omega}\right] .
$$

We claim that $\bar{f}$ is jointly u.s.c., where on $\mathscr{D}$ we consider the topology induced by $\widetilde{\Theta} \times \mathscr{P}_{L}$, with $\widetilde{\Theta}$ endowed with its product topology. Then let $\left|\vartheta^{n}-\vartheta^{*}\right|_{\infty} \rightarrow 0$ and $\mathbb{P}^{n} \rightarrow \mathbb{P}^{*}$. By Skorohod's representation, we have a probability space $(\widehat{\Omega}, \widehat{\mathscr{G}}, \widehat{\mathbb{P}})$ in which (A.5) holds true. It follows that

$$
\begin{aligned}
\limsup _{n \rightarrow \infty} \bar{f}\left(\vartheta^{n}, \mathbb{P}^{n}\right) & =\limsup _{n \rightarrow \infty} \mathbb{E}^{\widehat{\mathbb{P}}}\left[f\left(t^{n} \wedge \tau\left(\vartheta^{n}\right)+\mathrm{T}^{n}, \omega^{n} \otimes_{t^{n} \wedge \tau\left(\vartheta^{n}\right)} \mathrm{B}^{n}\right)\right] \\
& \leq \mathbb{E}^{\widehat{\mathbb{P}}}\left[\limsup _{n \rightarrow \infty} f\left(t^{n} \wedge \tau\left(\vartheta^{n}\right)+\mathrm{T}^{n}, \omega^{n} \otimes_{t^{n} \wedge \tau\left(\vartheta^{n}\right)} \mathrm{B}^{n}\right)\right] \\
& \leq \mathbb{E}^{\widehat{\mathbb{P}}}\left[f\left(t^{*} \wedge \tau\left(\vartheta^{*}\right)+\mathrm{T}^{*}, \omega^{*} \otimes_{t^{*} \wedge \tau\left(\vartheta^{*}\right)} \mathrm{B}^{*}\right)\right]=\bar{f}\left(\vartheta^{*}, \mathbb{P}^{*}\right),
\end{aligned}
$$

where the first inequality is due to Fatou's lemma and the second one is due to the $d_{\infty}$-u.s.c. of $f$.

Next note that

$$
V(\vartheta)=\sup _{\mathbb{P} \in \mathscr{P}_{L}(\tau, \vartheta)} \bar{f}(\vartheta, \mathbb{P}) .
$$

Taking into account that, by Lemma 4.2, $\mathscr{D}$ is closed, and considering the upper semicontinuity of $\bar{f}$, we can then apply Proposition 7.33, p. 153 in [1], to get a Borel-measurable kernel $\bar{v}: \widetilde{\Theta} \rightarrow \mathscr{P}$ such that $\bar{v}(\vartheta) \in \mathscr{P}_{L}(\tau, \vartheta)$ for all $\vartheta \in \widetilde{\Theta}$ and such that (A.7) holds true if $v$ is replaced by $\bar{v}$. Now we define $v(\vartheta):=\bar{v}\left(\vartheta_{t \wedge \tau(\vartheta) \wedge}\right.$. $)$, for all $\vartheta \in \widetilde{\Theta}$. Notice that the map $\vartheta \mapsto \vartheta_{t \wedge \tau(\vartheta) \wedge}$. is measurable from $\widetilde{\Theta}$ endowed with the sigma-algebra $\mathscr{G}_{\mathrm{T} \wedge \tau}$ into $\widetilde{\Theta}$ endowed with the Borel sigma-algebra $\mathscr{G}_{T}$. Hence the Borel measurability of $v$ entails the $\mathscr{G}_{\mathrm{T} \wedge \tau}$-measurability of $v$. Moreover, we have $V(\vartheta)=$ $V\left(\vartheta_{t \wedge \tau(\theta) \wedge}\right.$. $)$ by the very definition of $V$ and $\rho(\vartheta)=\rho\left(\vartheta_{\rho(\vartheta) \wedge}\right.$. $)$ for any stopping time $\rho$. It follows

$$
V(\vartheta)=V\left(\vartheta_{t \wedge \tau(\vartheta) \wedge .}\right)=\mathbb{E}^{\bar{v}\left(\vartheta_{t \wedge \tau(\vartheta) \wedge}\right)}\left[f^{t \wedge \tau\left(\vartheta_{t \wedge \tau(\vartheta) \wedge} \cdot\right), \omega_{t \wedge \tau(\vartheta) \wedge}}\right]=\mathbb{E}^{v(\vartheta)}\left[f^{t \wedge \tau(\vartheta), \omega}\right],
$$

which concludes the proof.

Let $\mathbb{P} \in \mathscr{P}_{L}, \tau$ be a continuous stopping time, and $v: \widetilde{\Theta} \rightarrow \mathscr{P}$ be a $\mathscr{G}_{\mathrm{T} \wedge \tau}$-measurable kernel such that $v(\vartheta) \in \mathscr{P}_{L}(\tau, \vartheta)$ for $\mathbb{P}$-a.e. $\vartheta \in \widetilde{\Theta}$, and define the concatenation:

$$
\mathbb{P} \otimes_{\tau} v(A)=\iint\left(1_{A}\right)^{\tau, \vartheta}\left(\vartheta^{\prime}\right) v\left(d \vartheta^{\prime} ; \vartheta\right) \mathbb{P}(d \vartheta), \quad A \in \mathscr{G}_{T} .
$$

Lemma A.4. Given a measurable function $f: \Theta \rightarrow R$ bounded from above, we have

$$
\mathbb{E}^{\mathbb{P}}\left[\mathbb{E}^{v(\mathrm{X})}\left[f^{\theta}\right]_{\mid \theta=(\mathrm{T} \wedge \tau, \mathrm{B})}\right]=\mathbb{E}^{\mathbb{P} \otimes_{\tau} v}[f] .
$$

Moreover, we have $\mathbb{P} \otimes_{\tau} v \in \mathscr{P}_{L}$.

Proof. The first statement follows from the definition (A.8) if $f$ is the indicator function $\mathbf{1}_{A}$. The general case follows by a standard approximation procedure.

Now the fact $\mathscr{P} \otimes_{\tau} v \in \mathscr{P}_{L}$ follows easily by considering that $\mathbb{P} \in \mathscr{P}_{L}$, that $v$ takes values in $\mathscr{P}_{L}$, and by using (A.8).

Finally, we prove the dynamic programming result. 
Proof of Proposition 4.3. By definition of nonlinear expectation and by the fact that any $\mathscr{P}_{L}(\tau, \vartheta)$ contains the dirac measure in $\vartheta=0$, we immediately have the inequality

$$
\overline{\mathscr{E}}_{L}\left[f 1_{\{\mathrm{T}<\tau\}}+\overline{\mathscr{E}}_{L}^{\tau}\left[f^{\theta}\right]_{\mid \theta=(\tau, \mathrm{B})} 1_{\{\tau \leq \mathrm{T}\}}\right] \geq \overline{\mathscr{E}}_{L}[f] .
$$

Regarding the converse inequality, we first note that, by Proposition A.3, there is a $\mathscr{G}_{t \wedge \tau}$-measurable kernel $v: \widetilde{\Theta} \rightarrow \mathscr{P}$ such that

$$
\overline{\mathscr{E}}_{L}^{\tau}\left[f^{t \wedge \tau, \omega}\right](\vartheta)=\mathbb{E}^{v(\vartheta)}\left[f^{t \wedge \tau, \omega}\right] .
$$

Therefore

$$
\mathbb{E}^{\mathbb{P}}\left[\overline{\mathscr{E}}_{L}^{\tau}\left[f^{\theta}\right]_{\mid \theta=(\tau, \mathrm{B})} 1_{\{\tau \leq \mathrm{T}\}}\right]=\mathbb{E}^{\mathbb{P}}\left[\overline{\mathscr{E}}_{L}^{\tau}\left[f^{\theta}\right]_{\mid \theta=(\mathrm{T} \wedge \tau, \mathrm{B})} 1_{\{\tau \leq \mathrm{T}\}}\right]=\mathbb{E}^{\mathbb{P}}\left[\mathbb{E}^{\nu(\mathrm{X})}\left[f^{\theta}\right]_{\mid \theta=(\mathrm{T} \wedge \tau, \mathrm{B})} 1_{\{\tau \leq \mathrm{T}\}}\right] .
$$

Now observe that if any function $g: \widetilde{\Theta} \rightarrow \mathbb{R}$ is $\mathscr{G}_{\top \wedge \tau}$-measurable, then $g^{\top \wedge \tau, \vartheta}(\cdot)$ is constantly equal to $g\left(\vartheta_{T \wedge \tau \wedge}.\right)$. By applying this fact to the function $g=\mathbf{1}_{\tau \leq T}(\mathrm{~T}, \mathrm{~B})$ and recalling Remark 2.3, it follows from Lemma A.4 that for any $\mathbb{P} \in \mathscr{P}_{L}$

$$
\mathbb{E}^{\mathbb{P}}\left[\mathbb{E}^{v(\mathrm{X})}\left[f^{\theta}\right]_{\theta=(\mathrm{T} \wedge \tau, \mathrm{B})} 1_{\{\tau \leq \mathrm{T}\}}\right]=\mathbb{E}^{\mathbb{P} \otimes_{\tau} v}\left[f 1_{\{\tau \leq \mathrm{T}\}}\right] .
$$

By applying the same observation to the $\mathscr{G}_{\mathrm{T} \wedge \tau}$-measurable function $f(\mathrm{~T}, \mathrm{~B}) \mathbf{1}_{\mathrm{T}<\tau}(\mathrm{T}, \mathrm{B})$ and by combining (A.9) and (A.10) with Lemma A.4, we obtain

$$
\mathbb{E}^{\mathbb{P}}\left[f 1_{\{\mathrm{T}<\tau\}}+\overline{\mathscr{E}}_{L}^{\tau}\left[f^{\theta}\right]_{\mid \theta=(\tau, \mathrm{B})} 1_{\{\tau \leq \mathrm{T}\}}\right]=\mathbb{E}^{\mathbb{P}}\left[\mathbb{E}^{v(\mathrm{X})}\left[f^{\theta}\right]_{\mid \theta=(\mathrm{T} \wedge \tau, \mathrm{B})}\right]=\mathbb{E}^{\mathbb{P} \otimes_{\tau} v}[f] \leq \overline{\mathscr{E}}_{L}[f],
$$

which shows the desired inequality. Now (4.2) follows by setting $\tau=\mathrm{T}$ in (4.1).

To prove the last part of the proposition, let $\mathbb{P}^{*} \in \mathscr{P}_{L}$ be an optimal probability measure such that $\overline{\mathscr{E}}_{L}[f]=\mathbb{E}^{\mathbb{P}^{*}}[f]$. It follows from the definition of nonlinear expectation and (4.2) that

$$
\overline{\mathscr{E}}_{L}[f]=\mathbb{E}^{\mathbb{P}^{*}}[f] \leq \mathbb{E}^{\mathbb{P}^{*}}\left[\overline{\mathscr{E}}_{L}^{\top}\left[f^{\top, \mathrm{B}}\right]\right] \leq \overline{\mathscr{E}}_{L}\left[\overline{\mathscr{E}}_{L}^{\top}\left[f^{\theta}\right]_{\mid \theta=(\mathrm{T}, \mathrm{B})}\right] \leq \overline{\mathscr{E}}_{L}[f]
$$

Therefore, $\mathbb{E}^{\mathbb{P}^{*}}[f]=\mathbb{E}^{\mathbb{P}^{*}}\left[\overline{\mathscr{E}}_{L}^{\mathrm{T}}\left[f^{\theta}\right]_{\mid \theta=(\mathrm{T}, \mathrm{B})}\right]$. Since we always have $f \leq \overline{\mathscr{E}}_{L}^{\mathrm{T}}\left[f^{\mathrm{T}, \mathrm{B}}\right]$, we finally obtain $f=\overline{\mathscr{E}}_{L}^{\top}\left[f^{\mathrm{T}, \mathrm{B}}\right], \mathbb{P}^{*}$-a.s.

We conclude this Appendix by showing the compactness of the operator $(A-I)^{-1}$ appearing in Section 7 and providing the proof of Proposition 7.2.

Proposition A.5. $(A-I)^{-1}$ is compact.

Proof. Let $\left\{x^{n}\right\}_{n \in \mathbb{N}} \subset H$ be a sequence weakly convergent to $x$ in $H$. To prove that $(A-I)^{-1}$ is compact it is sufficient to show that $(A-I)^{-1} x^{n} \rightarrow(A-I)^{-1} x$ strongly in $H$. Clearly $x_{0}^{n} \rightarrow x_{0}$ in $\mathbb{R}^{m}$ and $x_{1}^{n} \rightarrow x_{1}$ weakly in $H^{\prime}$. By looking at (7.4), to show that $\left|(A-I)^{-1}\left(x^{n}-x\right)\right|_{H}=\left|x^{n}-x\right|_{B} \rightarrow 0$, it is then sufficient to show that

$$
\lim _{n \rightarrow \infty} \int_{-\infty}^{0}\left|\int_{t}^{0} e^{-(s-t)}\left(x_{1}^{n}(s)-x_{1}(s)\right) d s\right|^{2} d t=0 .
$$

Since $x_{1}^{n} \rightarrow x_{1}$ weakly, we have

$$
\lim _{n \rightarrow \infty} \int_{t}^{0} e^{-(s-t)}\left(x_{1}^{n}(s)-x_{1}(s)\right) d s=0 \quad \forall t \in(-\infty, 0) .
$$

By Hölder's inequality and by boundedness of $\left\{x_{1}^{n}\right\}_{n \in \mathbb{N}}$ in $H^{\prime}$, we see that

$$
\sup _{\substack{t \in(-\infty, 0) \\ n \in \mathbb{N}}}\left|\int_{t}^{0} e^{-(s-t)}\left(x_{1}^{n}(s)-x_{1}(s)\right) d s\right|<\infty .
$$

By (A.12), (A.13), and Lebesgue's dominated convergence theorem, we obtain (A.11). 
Proof of Proposistion 7.2. The fact that (7.5) is continuous in case $f \in W^{1,2}((0, T), \mathbb{R})$ follows by Lemma 7.1 and by the discussion in [29, Remark 2.6], or directly by integration by part and Hölder's inequality.

We now prove the converse. We assume $m=1$; the proof for $m>1$ goes along the same lines. Let $f \in L^{2}((0, T), \mathbb{R})$ be such that (7.5) is $d_{B}$-continuous. We want to show that $f \in W^{1,2}((0, T), \mathbb{R})$. Let $V$ denote the subspace of $\mathbb{R} \times L^{2}\left(\mathbb{R}_{-}, \mathbb{R}\right)$ whose elements are the pairs $x=\left(x_{0}, x_{1}\right)$ where $x_{1}$ is continuous on $[-T, 0]$ and $x_{0}=x_{1}(0)$. By Lemma 7.1 we have that the linear functional

$$
\left(V,|\cdot|_{B}\right) \rightarrow \mathbb{R},\left(x_{0}, x_{1}\right) \mapsto \int_{-\infty}^{0} f(-s) \mathbf{1}_{\{s>-T\}} x_{1}(s) d s
$$

is continuous. We can then extend it to a continuous linear functional $\Lambda$ on the Hilbert space completion $\left(H_{B},|\cdot|_{B}\right)$ of $\left(V,|\cdot|_{B}\right)$, which coincides with the completion of $\left(H,|\cdot|_{B}\right)$ because $V$ is $|\cdot|_{H}$-dense in $H$ and $|\cdot|_{B}$ is weaker than $|\cdot|_{H}$.

By the Riesz representation theorem it then follows that there exists $z \in H_{B}$ such that

$$
\Lambda x=\langle z, x\rangle_{B} \quad \forall x \in H_{B} .
$$

By definition of $|\cdot|_{B}, A-I:\left(D(A),|\cdot|_{H}\right) \rightarrow\left(H,|\cdot|_{B}\right)$ is an isometry and then it can be uniquely extended to its closure $\overline{A-I}:\left(H,|\cdot|_{H}\right) \rightarrow\left(H_{B},|\cdot|_{B}\right)$ (we refer to [29] for further details). Then we can write

$$
\langle z, x\rangle_{B}=\left\langle(\overline{A-I})^{-1} z,(\overline{A-I})^{-1} x\right\rangle_{H}=\left\langle(\overline{A-I})^{-1} z,(A-I)^{-1} x\right\rangle_{H}=\left\langle\left(A^{*}-I\right)^{-1}(\overline{A-I})^{-1} z, x\right\rangle_{H} \quad \forall x \in H .
$$

Then, we obtain that there exists $y:=\left(A^{*}-I\right)^{-1}(\overline{A-I})^{-1} z$ such that

$$
\langle y, x\rangle_{H}=\Lambda x \quad \forall x \in H .
$$

Since $y \in D\left(A^{*}\right)=\mathbb{R} \times W^{1,2}\left(\mathbb{R}_{-}\right)$and since (A.14) holds in particular for all $x \in V$, we conclude $f(-\cdot) \in W^{1,2}((-T, 0), \mathbb{R})$.

\section{References}

[1] Dimitri P. Bertsekas and Steven E. Shreve. Stochastic optimal control: the discrete time case. Elsevier Science, 1979.

[2] R. Cont and D. Fournié. Functional Itô calculus and stochastic integral representation of martingales. Ann. Probab., 41:109-133, 2013.

[3] A. Cosso, S. Federico, F. Gozzi, M. Rosestolato, and N. Touzi. Path-dependent equations and viscosity solutions in infinite dimension. Ann. Probab., 46(1):126-174, 2018.

[4] M. G. Crandall, H. Ishii, and P. L. Lions. User's guide to viscosity solutions of second order partial differential equations. Bulletin of the American mathematical society, 27(1):1-67, 1992.

[5] M. G. Crandall and P. L. Lions. Viscosity solutions of Hamilton-Jacobi equations. Trans. Amer. Math. Soc., 277:1-42, 1983.

[6] B. Dupire. Functional Itô calculus. papers.ssrn.com, 2009.

[7] I. Ekren. Viscosity solutions of obstacle problems for fully nonlinear path-dependent PDEs. Stochastic Processes and their Applications, 127(12):3966-3996, 2017. 
[8] I. Ekren, C. Keller, N. Touzi, and J. Zhang. On viscosity solutions of path-dependent PDEs. Ann. Probab., 42:204-236, 2014.

[9] I. Ekren, N. Touzi, and J. Zhang. Optimal stopping under nonlinear expectation. Stochastic Processes and their Applications, 124(10):3277-3311, 2014.

[10] I. Ekren, N. Touzi, and J. Zhang. Viscosity solutions of fully nonlinear parabolic pathdependent PDEs: Part I. Ann. Probab., 44:1212-1253, 2016.

[11] I. Ekren, N. Touzi, and J. Zhang. Viscosity solutions of fully nonlinear parabolic pathdependent PDEs: Part II. Ann. Probab., 44:2507-2553, 2016.

[12] G. Fabbri, F. Gozzi, and A. Świech. Stochastic Optimal Control in Infinite Dimensions. Springer, 2017.

[13] H. Federer. Geometric measure theory. Springer, 1969.

[14] A. Kalinin and A. Schied. Mild and viscosity solutions to semilinear parabolic pathdependent PDEs. Preprint arXiv:1611.08318.

[15] I. Karatzas and S. E. Shreve. Brownian Motion and Stochastic Calculus. Springer, $2^{\text {nd }}$ edition, 1991.

[16] C. Keller. Viscosity solutions of path-dependent integro-differential qquations. Stochastic Processes and their Applications, 126(9):2665-2718, 2016.

[17] P.A. Meyer and W.A. Zheng. Tightness criteria for laws of semimartingales. Annales de l'I.H.P. Probabilitś et statistiques, 20(4):353-372, 1984.

[18] M. Nutz and R. van Handel. Constructing Sublinear Expectations on Path Space. Stochastic Processes and their Applications, 123(8):3100-3121, 2013.

[19] S. Peng. Backward stochastic differential equation, nonlinear expectation and their applications. Proceedings of the International Congress of Mathematicians, Hyderabad, India, 2010.

[20] S. Peng and F. Wang. BSDE, path-dependent PDE and nonlinear Feynman-Kac formula. Sci. China Math., 59:19-36, 2016.

[21] S. Peszat and J. Zabczyck. Stochastic partial differential equations with Lévy noise. An evolution equation approach. Cambridge University Press, 2007.

[22] T. Pham and J. Zhang. Two person zero-sum game in weak formulation and path-dependent bellman-isaacs equation. SIAM J. Control Optim., 52:2090-2121, 2014.

[23] Z. Ren. Viscosity solutions of fully nonlinear elliptic path dependent partial differential equations. Ann. Appl. Probab., 26(6):3381-3414, 2016.

[24] Z. Ren. Perron's method for viscosity solutions of semilinear path dependent PDEs. Stochastics, 89(6-7):843-867, 2017.

[25] Z. Ren and X. Tan. On the convergence of monotone schemes for path-dependent PDEs. Stochastic Processes and their Applications, 127(6):1738-1762, 2017.

[26] Z. Ren, N. Touzi, and J. Zhang. Comparison of viscosity solutions of semilinear pathdependent partial differential equations. Preprint, arXiv:1410.7281. 
[27] Z. Ren, N. Touzi, and J. Zhang. An overview on viscosity solutions of path-dependent PDEs. In D. Crisan, B. Hambly, and T. Zariphopoulou, editors, Stochastic Analysis and Applications 2014 - In Honour of Terry Lyons, Springer Proceedings in Mathematics and Statistics, pages 397-453, 2014.

[28] Z. Ren, N. Touzi, and J. Zhang. Comparison of viscosity solutions of fully nonlinear degenerate parabolic path-dependent PDEs. SIAM J. Math. Analysis, 49:4093-4116, 2017.

[29] M. Rosestolato and A. Świech. Partial regularity of viscosity solutions for a class of Kolmogorov equations arising from mathematical finance. J. Differential Equations, 262(3):1897-1930, 2017. 